\title{
OPEN Synthesis of nano-fibers containing nano-curcumin in zein corn protein and its physicochemical and biological characteristics
}

\author{
Narges Fereydouni ${ }^{1,2,3 凶}$, Jebrail Movaffagh ${ }^{4,5}$, Nafise Amiri $^{4,5}$, Susan Darroudi 6 , \\ Aida Gholoobi ${ }^{7}$, Arash Goodarzi ${ }^{1,2}$, Alireza Hashemzadeh ${ }^{3,8,9}$ \& Majid Darroudi ${ }^{3,10 凶}$
}

Curcumin contains many biological activities as a natural bioactive substance, however, its low solubility stands as a huge bioavailability disadvantage. Recently, different methods have been developed for utilizing the tremendous medicinal properties of this material. In this study, an Oil/ Water nano-emulsion of curcumin (Nano-CUR) has been woven in zein polymer at three percentages of $5 \%, 10 \%$, and $15 \%(\mathrm{v} / \mathrm{v})$. We have investigated the physicochemical properties of nanofibers (NFs) including FESEM, FTIR, tensile strength, encapsulation efficiency, and release profile, as well as biological properties. According to the data, the NFs have been observed to become significantly thinner and more uniformed as the involved percentage of Nano-CUR had been increased from 5 to $15 \%$. It is considerable that the tensile strength can be increased by heightening the existing NanoCUR from $5 \%$ towards $15 \%$. The resultant NFs of zein/Nano-CUR $15 \%$ have exhibited higher in vitro release and lower encapsulation efficiency than the other evaluated zein/Nano-CUR NFs. It has been confirmed through the performed viability and antioxidant studies that zein/Nano-CUR $10 \%$ NFs are capable of providing the best conditions for cell proliferation. Considering the mentioned facts, this work has suggested that Nano-CUR can be successfully woven in zein NFs and maintain their biological properties.

As a branch of nanomaterials, nano-fibers (NFs) have been widely used for biomedical and many other applications throughout the recent years due to their intrinsic properties ${ }^{1}$. Relatively, NFs are classified as carriers with a nanostructure diameter of less than $100 \mathrm{~nm}$, while the fibers that contain a diameter of less than $1000 \mathrm{~nm}$ are also included in this category; the measurement of these materials is commonly done by the employment of electrospinning technique. However, a larger surface can be constructed by reducing the diameter of fibers from micrometer to under micrometer or $\mathrm{nm}^{2}$.

NFs can be synthesized from natural and synthetic polymers or their different compositions. In addition, they can be efficiently delivered by both hydrophilic and hydrophobic drugs ${ }^{3,4}$. Different parameters can modulate the process of NFs drug release such as the ratio of drug to polymer, diameter, morphology, and/or NFs porosity ${ }^{2}$. Among the available methods for preparing $\mathrm{NFs}^{5-7}$, the electrospinning technique stands as the most accepted one since it has proved to be cost-effective and contain a high efficiency for producing these materials in the range of $\mathrm{nm}$ to micro-meters with varying molecules ${ }^{8}$.

\footnotetext{
${ }^{1}$ Department of Tissue Engineering, School of Medicine, Fasa University of Medical Sciences, Fasa, Iran. ${ }^{2}$ Noncommunicable Diseases Research Center, Fasa University of Medical Sciences, Fasa, Iran. ${ }^{3}$ Department of Medical Biotechnology and Nanotechnology, School of Medicine, Mashhad University of Medical Sciences, Mashhad, Iran. ${ }^{4}$ Department of Pharmaceutical Nanotechnology, School of Pharmacy, Mashhad University of Medical Sciences, Mashhad, Iran. ${ }^{5}$ Targeted Drug Delivery Research Center, Pharmaceutical Technology Institute, Mashhad University of Medical Sciences, Mashhad, Iran. ${ }^{6}$ Student Research Committee, International UNESCO Center for Health-Related Basic Sciences and Human Nutrition, Mashhad University of Medical Sciences, Mashhad, Iran. ${ }^{7}$ Medical Genetics Research Center, School of Medicine, Mashhad University of Medical Sciences, Mashhad, Iran. ${ }^{8}$ Department of Medical Physiology, Faculty of Medicine, Mashhad University of Medical Sciences, Mashhad, Iran. ${ }^{9}$ Metabolic Syndrome Research Center, Mashhad University of Medical Sciences, Mashhad, Iran. ${ }^{10}$ Nuclear Medicine Research Center, Mashhad University of Medical Sciences, Mashhad, Iran. ${ }^{\square}$ email: Narges.fereydouni2020@gmail.com; darroudim@mums.ac.ir
} 
Due to the encapsulation of drug molecules in a polymer structure, NFs can function as a drug delivery system, be applicable for skin treatments, and contribute to simple releases that take place throughout topical and systemic procedures ${ }^{9}$. In order to provide a proper wound healing, several parameters are required to be considered such as air conditioning, preventing the entrance of microorganisms into wound bed, proper dressing, and adhesion to the wound site ${ }^{10}$. Hereof, NF-based systems can benefit this process in several ways such as facilitating better conditions for the cellular respiration through their high permeability and provide an ideal environment for the wounds to be repaired. The moisture that exists under wound dressing can help the healing procedure and supply a suitable barrier for the ulcerative exudates, which are responsible for enclosing proteins and protective cytokines in the wound. The rate of vapor transmission through the wound stands as another significant parameter for choosing the proper materials ${ }^{11-13}$.

Plant proteins are known to be low immunogenic, low-cost, affordable, and highly biocompatible, which are commonly extracted from plants or the byproducts of agricultural industry ${ }^{14}$. Some of these proteins, such as soy protein, zein (corn), and gluten (wheat), are being currently used in food packaging industry, while their potential for being applied in health applications is being examined as well. Being a corn protein, zine is a byproduct of bioethanol industry that has been widely studied in regards to the biomedical applications ${ }^{15}$. This particular material contains certain valuable properties such as hardness, flexibility, hydrophobicity, anti-bacterial resistance, and anti-oxidant activity ${ }^{15-17}$. Miyoshi et al., have initially employed the technique of electrospinning for the purpose of synthesizing NFs and thereafter, investigated the existing relationship between the inverse ratio of polymer concentration and electric potential energy, as well as their effects on the properties of NFs. The concentration of zein solution that had been applied in the synthesis of NF has been evaluated at $18-60 \%{ }^{18}$. It has been indicated by the work of Torres-Giner et al. that the acidic solution of zein can produce smoother NFs, while the alkaline solution leads to the formation of mold-fiber beaded structures ${ }^{19}$.

By the usage of NFs based systems, various natural and synthetic materials have been investigated for wound healing throughout the recent years. Curcumin is a natural polyphenolic material that is extracted from the root of Curcuma longa (Zingiberaceae family) and has been widely utilized as a medicinal plant in many Asian countries over the past years ${ }^{20}$. It is applied in traditional medicine for the treatment of different diseases such as anorexia, cough, and biliary problems, as well as liver and sinus illnesses ${ }^{21}$. Many studies have reported the antibacterial, anti-proliferation, anti-inflammatory, anti-oxidant, anti-cancer, and anti- amylogenic properties of curcumin in cellular and animal models ${ }^{22-24}$. Other activities that have been assigned to curcumin include antirheumatic, anti-viral, liver, anti-HIV, and wound healing features ${ }^{25}$. Furthermore, curcumin and tumeric have been approved as safe products by the FDA, FAO, and WHO organizations ${ }^{26}$. There has not been any reported side effects from the oral administration of this material in doses that reach up to $12 \mathrm{~g}$ per day throughout the clinical trials ${ }^{27}$.

Curcumin is a hydrophobic polyphenol that contains certain properties in low degrees, such as low solubility in water, low stability, rapid metabolism, low absorption, and availability, which have reduced its medical benefits in great extents ${ }^{28}$. To overcome these obstacles, drug delivery systems have been considered since they can prolong the flow time, permeability, and resistance towards metabolic degradation ${ }^{29-31}$. One of these methods would be the determination of synthesized curcumin in a scaffold bed of NFs, while up to the present, this material has been woven in the NFs mats that are composed of different polymers ${ }^{32-43}$. Researchers have prepared various wound dressing nanocomposites that had been consisted of curcumin-NFs in order to understand the mechanism of repairing diabetic ulcers, burns, and etc. by curcumin-loaded NFs, examine the physicochemical properties and release of curcumin from the polymer bed, and investigate the process of wound healing. It has been suggested by the obtained results that the process of wound healing with curcumin-NFs may occur by preserving the antioxidant, anti-inflammatory, and anti-bacterial properties of curcumin, as well as the inherent properties of polymer scaffolds ${ }^{44}$. However, there are still limitations to the solubility and stability of curcumin although many methods such as liposome ${ }^{45}$, polymeric nanoparticles ${ }^{46}$, solid lipid nanoparticles (SLNs) ${ }^{47}$, albumin nanoparticles ${ }^{48}$, microemulsions and nanoemulsions $s^{49}$, nanospheres, and microcapsules ${ }^{50}$ have been devised in recent years to obtain a higher aqueous solubility, bioavailability, activity, and lower toxicity ${ }^{28,51}$. In this regard, the emulsion method has received much attention in recent decades due to containing a simple synthesizing process and high efficiency, as well as requiring low costs, and eco-friendly materials ${ }^{52-56}$. This method has been exerted to synthesize water-insoluble substances ${ }^{57,58}$, oils ${ }^{59,60}$, plant extracts ${ }^{61,62}$, proteins ${ }^{63}$, nucleic acids ${ }^{64}$, curcumin, and many other substances ${ }^{49}$ for drug delivery intentions, while some of these materials have been evaluated even in nanofiber scaffolds ${ }^{65-67}$. According to the available data, there has not been any studies conducted on the usage of $\mathrm{O} / \mathrm{W}$ nano-emulsion of curcumin in nanofiber substrates. Therefore, we have attempted in this work to investigate the possibility of $\mathrm{O} / \mathrm{W}$ nano-emulsion curcumin (Nano-CUR) loading in zein nanofibers and evaluate the physicochemical properties, release, and cytotoxicity of zein/Nano-CUR NFs.

\section{Results}

Morphological study. The morphology of electrospun NFs has been the first feature of these membrane models that had been aimed to be investigated. In this study, the Nano-CUR, which had been successfully prepared in our previous work, has been loaded in zein polymer through the means of electrospinning method while applying the appropriate parameters including solvent, the concentrations of final polymer and NanoCUR, and flow speed. Figure 1 demonstrates the FESEM micrographs of zein NFs that had contained the varying Nano-CUR volumes of 5\%,10\%, and $15 \%(\mathrm{v} / \mathrm{v})$. According to our observations, the existing NFs have become significantly thinner and more uniformed as the percentage of Nano-CUR had been increased from 5 to $15 \%$. It has been also indicated that Nano-CUR had been thoroughly mixed with the polymer solutions due to the lack of detecting any Nano-CUR aggregates throughout the surface of NF. 
A

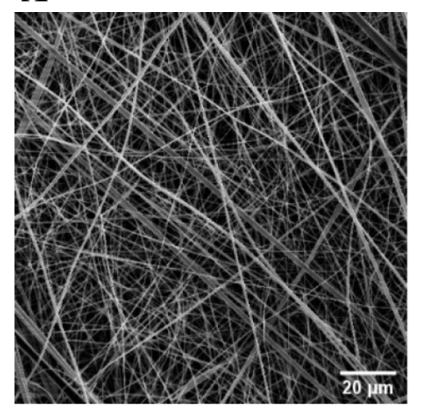

B

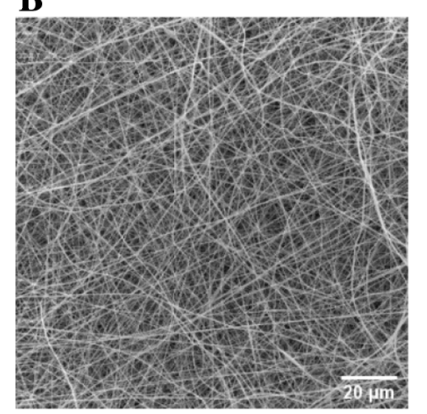

C

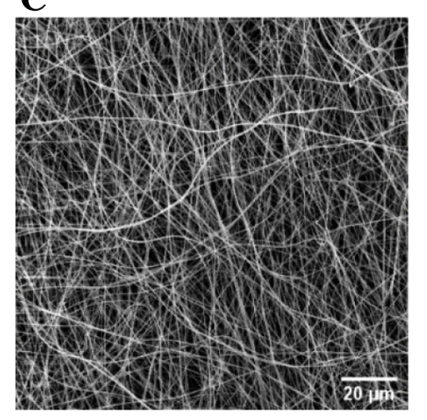

D

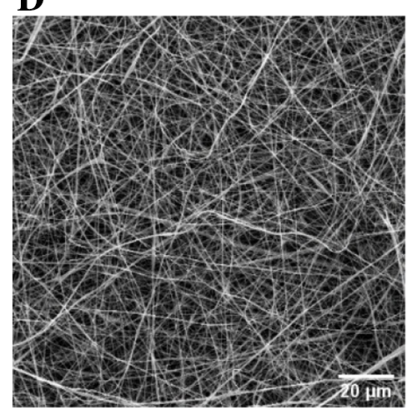

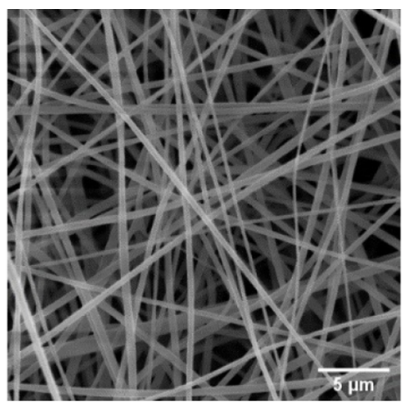
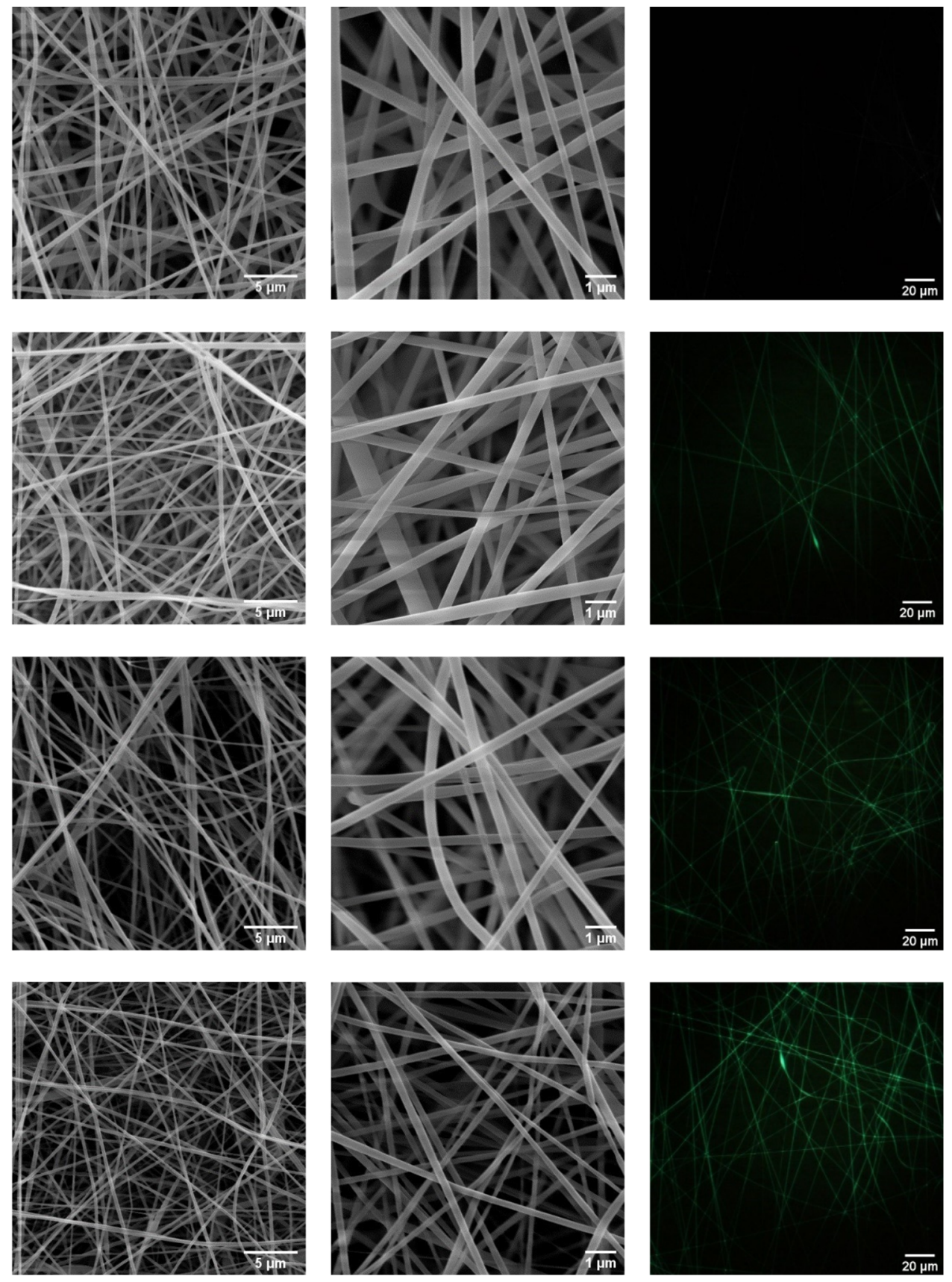

Figure 1. FESEM images of (A) zein NFs, (B) zein/Nano-CUR 5\% NFs, (C) zein/Nano-CUR 10\% NFs, (D) zein/Nano-CUR 15\% NFs.

As it is displayed in Fig. 2 and Table 1, the mean diameter and diameter distribution of NFs have faced a significant reduction upon the addition of Nano-CUR to the zein solution. However, it has been perceived in the obtained outcomes that the electrospinning of zein/Nano-CUR solution in a similar volume has resulted in different thicknesses of NFs. Furthermore, the thicknesses and porosity percentages of zein/Nano-CUR $10 \%$ $(p<0.05)$ and $15 \%$ NFs $(p<0.001)$ have been significantly higher than that of the zein blank NFs $(p<0.01)$. In addition, the viscosity of as-prepared zein/Nano-CUR 5\%,10\%, and 15\% solutions has faced a notable decrease when compared to the zein solution $(p<0.001)$.

We have observed the fluorescence of nanofibers through the application of a fluorescent microscope (DP73) (Fig. 1), while the fluorescent emission of zein nanofibers had been collected within the range of green light wavelength. Zein NFs and zein/Nano-CUR NFs have exhibited green fluorescent signals without using any external fluorescent dyes, however, the intensity of fluorescent has been significantly increased as the volume of Nano-CUR had been enlarged. Next to discovering the even distribution of Nano-CUR throughout the nanofibers, we have indicated their lack of accumulation by observing the uniformed fluorescent signals. These results 

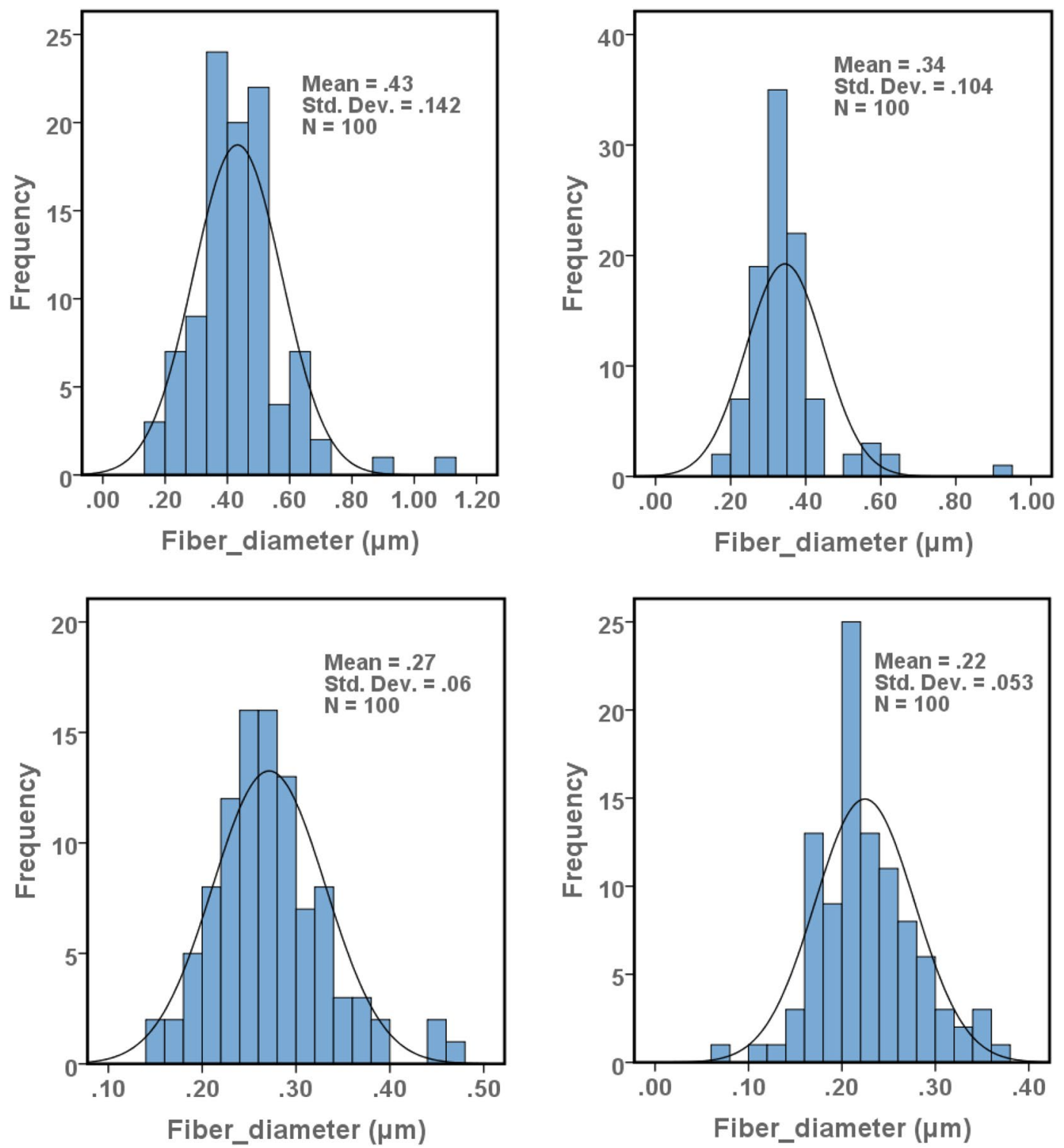

Figure 2. Size distribution of (A) zein NFs, (B) zein/Nano-CUR 5\% NFs, (C) zein/Nano-CUR 10\% NFs, (D) zein/Nano-CUR 15\% NFs.

\begin{tabular}{|l|c|l|l|c|l|}
\hline & Zein NF & $\begin{array}{l}\text { Zein/Nano-CUR } \\
\mathbf{5 \% ~ N F}\end{array}$ & $\begin{array}{l}\text { Zein/Nano-CUR } \\
\mathbf{1 0 \% ~ N F}\end{array}$ & $\begin{array}{l}\text { Zein/Nano-CUR } \\
\mathbf{1 5 \%} \text { NF }\end{array}$ & Open container \\
\hline Diameter $(\mathrm{nm})$ & $430 \pm 142$ & $340 \pm 104^{\mathrm{a}}$ & $270 \pm 60^{\mathrm{ab}}$ & $220 \pm 53^{\mathrm{abc}}$ & \\
\hline Thickness $(\mathrm{mm})$ & $0.082 \pm 0.01$ & $0.0964 \pm 0.01$ & $0.112 \pm 0.01^{\mathrm{a}}$ & $0.133 \pm 0.02^{\mathrm{ab}}$ & \\
\hline Viscosity (Pa.s) & $0.534 \pm 0.03$ & $0.373 \pm 0.04^{\mathrm{a}}$ & $0.274 \pm 0.02^{\mathrm{ab}}$ & $0.267 \pm 0.02^{\mathrm{ab}}$ & \\
\hline Porosity $(\%)$ & $28.3 \pm 6.5$ & $33.2 \pm 4.5$ & $34.6 \pm 6.4^{\mathrm{a}}$ & $35.2 \pm 2.4^{\mathrm{a}}$ & \\
\hline WVTR $\left(\mathrm{g} / \mathrm{m}^{2}\right)$ & $1680.64 \pm 248.34$ & $1840.00 \pm 290.35$ & $2005.26 \pm 250.36$ & $2550.27 \pm 246.25^{\mathrm{ab}}$ & $5355.25 \pm 357.28$ \\
\hline Water-uptake capacity & $29.87 \pm 1.57$ & $32.23 \pm 2.12$ & $35.64 \pm 3.14^{\mathrm{a}}$ & $37.34 \pm 2.15^{\mathrm{ab}}$ & \\
\hline
\end{tabular}

Table 1. Physicochemical properties of zein and zein/Nano-CUR 5\%, 10\%, and 15\% NFs. Data displayed as mean \pm SD. a: significant difference between zein/Nano-CUR $5 \%, 10 \%$, and $15 \%$ NFs in comparison with zein NF, b: significant difference between zein/Nano-CUR 10\%, and 15\% NFs in comparison with zein/Nano-CUR $5 \%$ NF, c: significant difference between zein/Nano-CUR 15\% NFs in comparison with zein/Nano-CUR 10\% NF. 


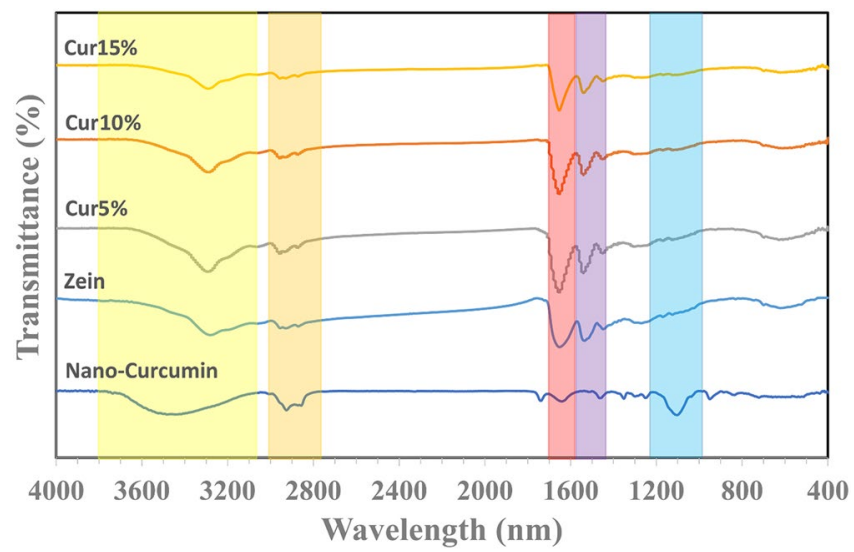

Figure 3. FTIR image of Nano-CUR, zein NFs, zein/Nano-CUR 5\% NFs, zein/Nano-CUR 10\% NFs, zein/ Nano-CUR 15\% NFs.
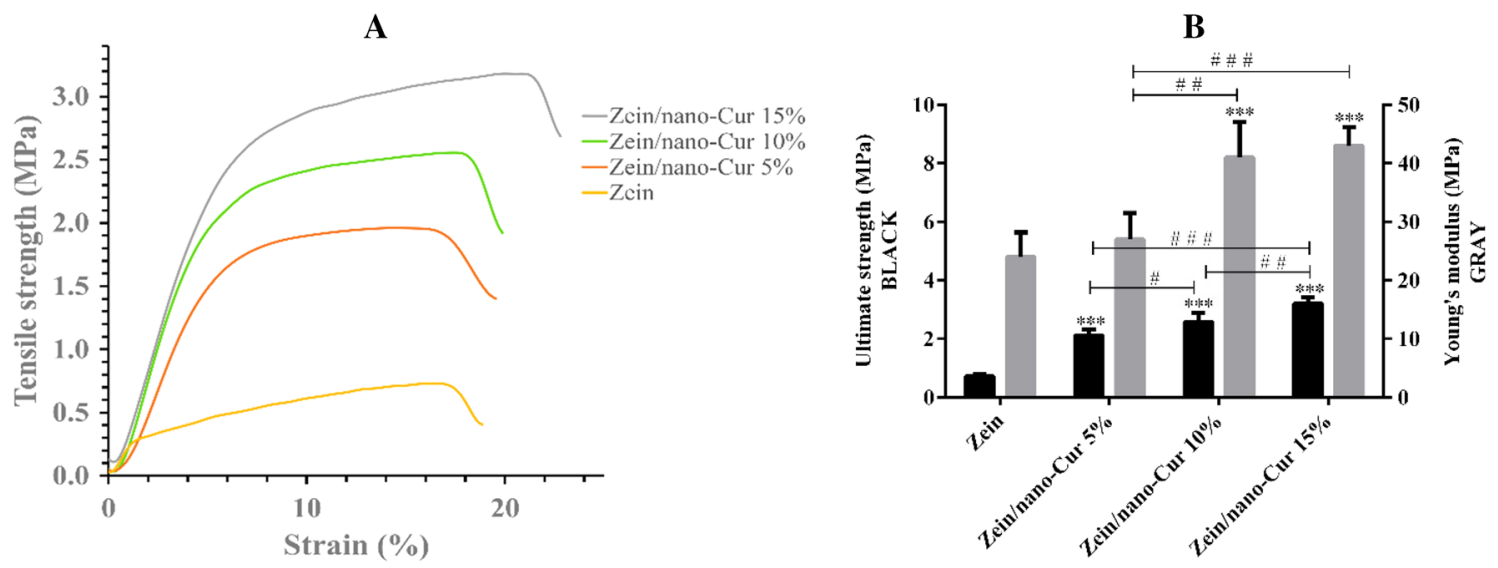

Figure 4. The stress-strain diagram (A) and the ultimate strength and young's modulus parameters $(\mathbf{B})$ of zein and zein/Nano-CUR 5\%, 10\%, and 15\% NFs; \# indicates significance between groups and * indicates significance of every group with zein group $\left(\left(^{\# / *} p<0.05,{ }^{\# \# / * *} p<0.01\right.\right.$, and $\left.{ }^{\# \# \# / * * *} p<0.001\right)$.

are suggestive of Nano-CUR high compatibility with zein polymer and the formation of a homogeneous solution even in the course of spinning step.

FTIR study. Figure 3 illustrates the FTIR spectra of Nano-CUR, zein, and zein/Nano-CUR 5\%, 10\%, and $15 \%$ NFs, which had been obtained to determine the inter-molecular interactions and composition. The structure of zein NFs has been characterized by the utilization of amide vibrations at $1650 \mathrm{~cm}^{-1}$ (amide I) and $1536 \mathrm{~cm}^{-1}$ (amide II) ${ }^{68}$. The available spectrum of Nano-CUR, which had been previously pointed out at $3484 \mathrm{~cm}^{-1}, 2925 \mathrm{~cm}^{-1}$, and $1103 \mathrm{~cm}^{-1}$, has been related to the existing vibration stretching of O-H, C-H, and C-O groups ${ }^{49,69}$. In regards to the case of zein/Nano-CUR NFs, we have detected the appearances of typical amide I and II bands at around 1650 and $1536 \mathrm{~cm}^{-1}$, respectively.

Mechanical measurement. We have assessed the mechanical properties of zein and zein/Nano-CUR NFs, which had included Young's modulus, elongation percentage at break point, and tensile strength. Figure 4A depicts the stress-strain diagrams of zein and zein/Nano-CUR NF scaffolds. The ultimate tensile strength of zein and zein/Nano-CUR 5\%, 10\%, and 15\% NFs have been $0.73 \pm 0.06,2.11 \pm 0.2,2.58 \pm 0.3$, and 3.21 $\pm 0.21 \mathrm{MPa}$, respectively. According to the results, the ultimate tensile strength of zein NFs has been significantly heightened as the amount of Nano-CUR had been increased $(p<0.001)$ (Fig. $4 \mathrm{~B})$. The young's modulus of zein and zein/Nano-CUR 5\%, $10 \%$, and $15 \%$ NFs have been measured to be $24 \pm 4.2,27 \pm 4.5,41 \pm 6.1$, and $43 \pm 3.2 \mathrm{MPa}$, respectively. The Young's modulus of zein/Nano-CUR $10 \%$ and $15 \%$ has been significantly increased in comparison to the zein NFs $(p<0.001)$ (Fig. 4B). Moreover, the elongation (\%) at break point have been detected to be $17.06 \% \pm 5.3 \%, 11.36 \% \pm 2.2 \%, 17.29 \% \pm 3.1 \%$, and $20.51 \% \pm 5.1 \%$, respectively, which had been indicative of the higher percentage of zein/Nano-CUR 15\% NF in comparison to that of the zein/Nano-CUR 5\% NF $(p<0.05)$. The obtained outcomes from the performed mechanical examination have suggested that increasing the portion of Nano-CUR can result in extending the mechanical properties of NFs. 


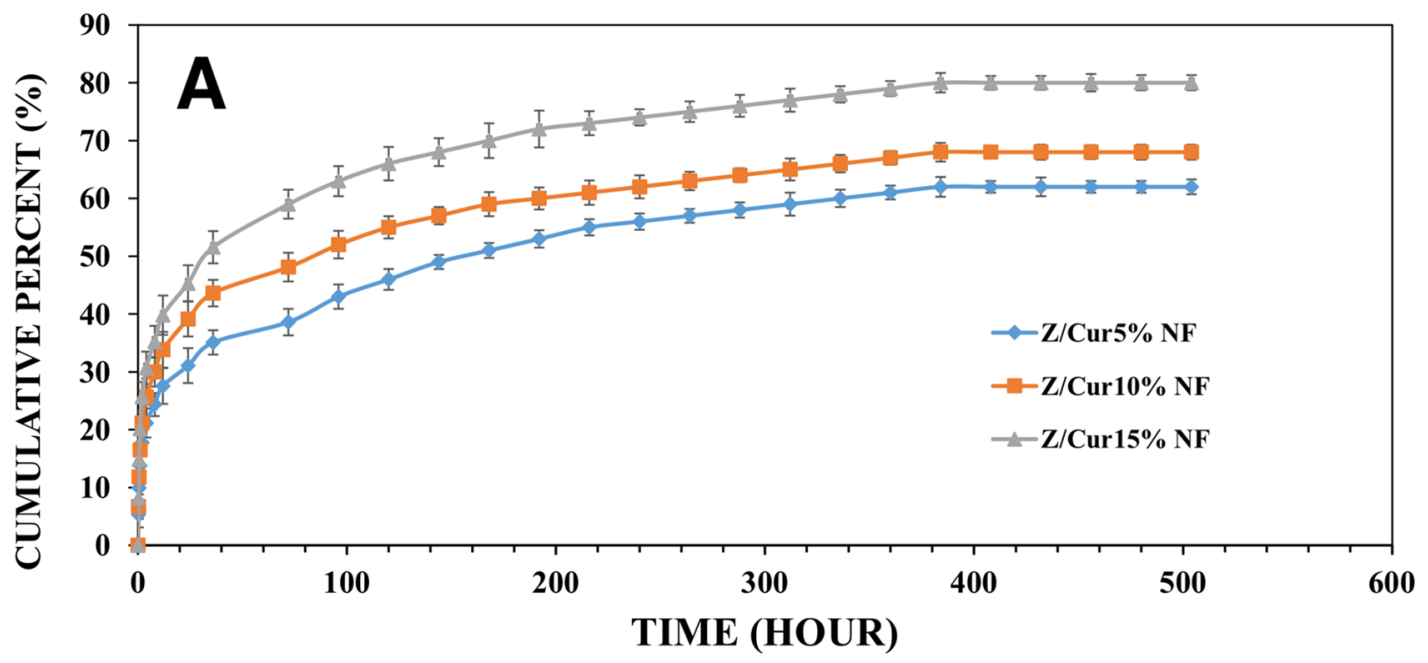

B

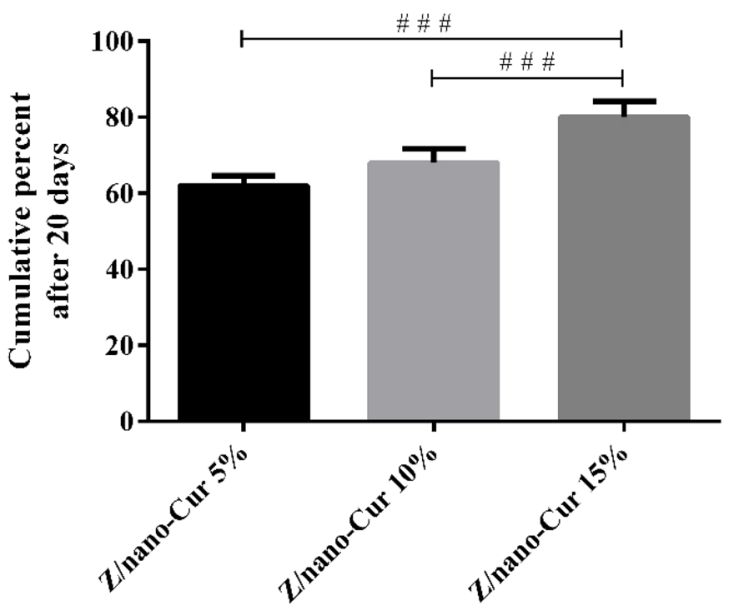

C

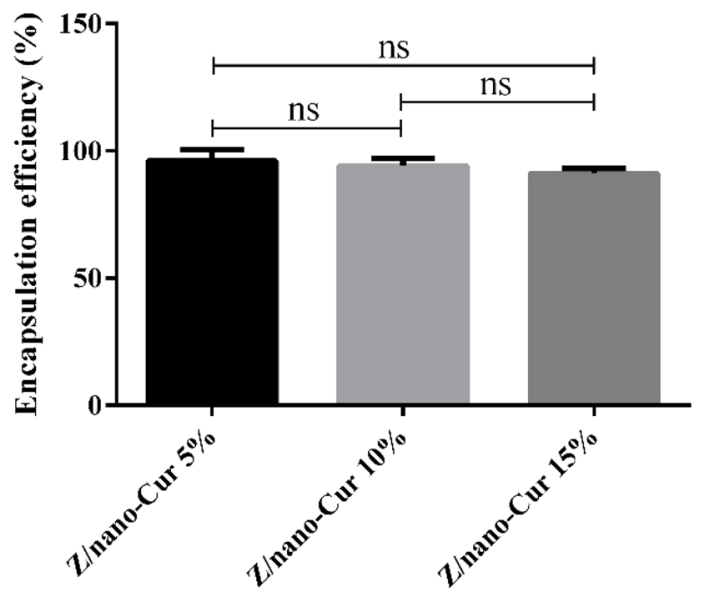

Figure 5. The release diagram of Nano-CUR $5 \%, 10 \%$, and $15 \%$ from zein NFs in 20 days (A), in vitro release percentage (B), and encapsulation efficiency diagrams (C) of zein/Nano-CUR 5\%, 10\%, and 15\% NFs; \# indicates significance between groups and ${ }^{*}$ indicates significance of every group with zein group $\left({ }^{* *} p<0.05\right.$, $\# \#<<0.01$, and $\left.{ }^{\# \# \# / * * *} p<0.001\right)$.

Drug EE\% and release assessment. The release profile of Nano-CUR that had been obtained from electrospun zein/Nano-CUR 5\%, 10\%, and 15\% NFs is demonstrated in Fig. 5A. Although the release models in regards to all of the involved zein/Nano-CUR 5\%,10\%, and 15\% NFs have been similar, yet the electrospun zein/Nano-CUR 15\% NFs had displayed a higher release rate than the zein/Nano-CUR 5\% and 10\% NFs. Zein/ Nano-CUR $5 \%, 10 \%$, and $15 \%$ have released $62 \% \pm 2.6 \%, 68 \% \pm 3.7 \%$, and $80 \% \pm 4.2 \%$ of Nano-CUR in 20 days, respectively. The observed significant difference between zein/Nano-CUR 15\% NFs, 5\% NFs $(p<0.001)$, and $10 \%$ NFs $(p<0.001)$ is exhibited in Fig. 5B. Zein/Nano-CUR 5\%, 10\%, and 15\% NFs has displayed a burst release of $40 \%, 34 \%$, and $28 \%$ at the first time interval of $12 \mathrm{~h}$, respectively, which had been continuous until the release became sustained and reached a plateau phase. According to Fig. 5C, the EE\% of zein/Nano-CUR 5\%, 10\%, and $15 \%$ NFs have been $96 \% \pm 4.42 \%, 94 \% \pm 3.04 \%$ and $91 \% \pm 2.01 \%$, respectively, without displaying any significant differences.

WVTR. The amount of water vapor transmission (WVTR) in $\mathrm{g} / \mathrm{m}^{2}$ regarding zein and zein/Nano-CUR nanofiber scaffolds of $5 \%, 10 \%$, and $15 \%$ are illustrated in Table 1 . The WVTR of zein and zein/Nano-CUR $5 \%, 10 \%$, and $15 \%$ have been $3220.64 \pm 248.34 \mathrm{~g} / \mathrm{m}^{2}, 2550.27 \pm 246.25 \mathrm{~g} / \mathrm{m}^{2}, 2005.26 \pm 250.36 \mathrm{~g} / \mathrm{m}^{2}$, and $1840.00 \pm 290.35 \mathrm{~g} / \mathrm{m}^{2}$, respectively. The performed statistical studies have indicated that the WVTR of zein NFs had been significantly higher than the scaffold of zein/Nano-CUR $10 \%$ and $15 \%$.

Water-uptake capacity. The amount of water-uptake capacity percentages of scaffolds in regards to zein and zein/Nano-CUR nanofiber scaffolds of 5\%,10\%, and $15 \%$ are presented in Table 1 . The water-uptake capacity percentages of zein and zein/Nano-CUR $5 \%, 10 \%$, and $15 \%$ have been measured to be $29.87 \pm 1.57 \%$, 


\section{A) HDF}

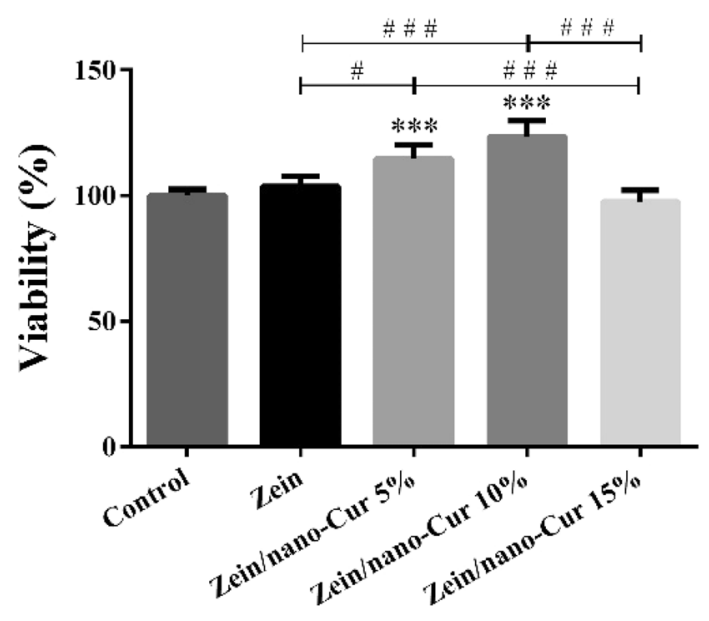

C) $\mathrm{HDF}$

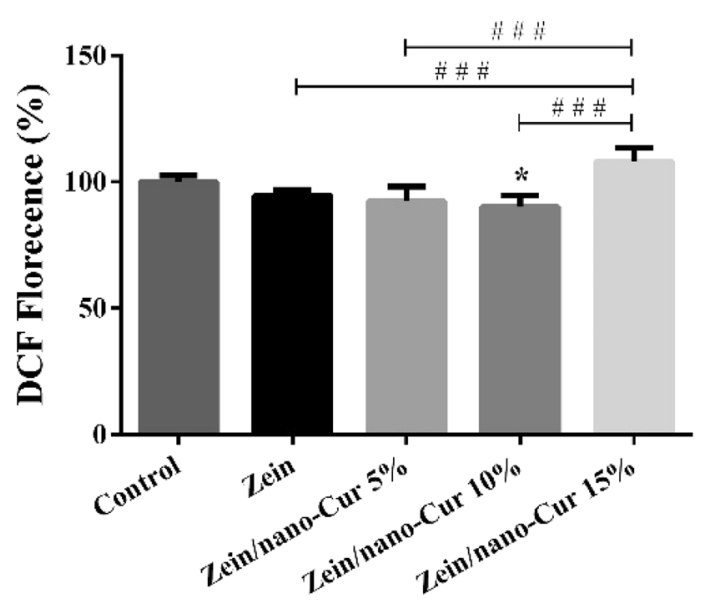

B) $\mathbf{L 9 2 9}$

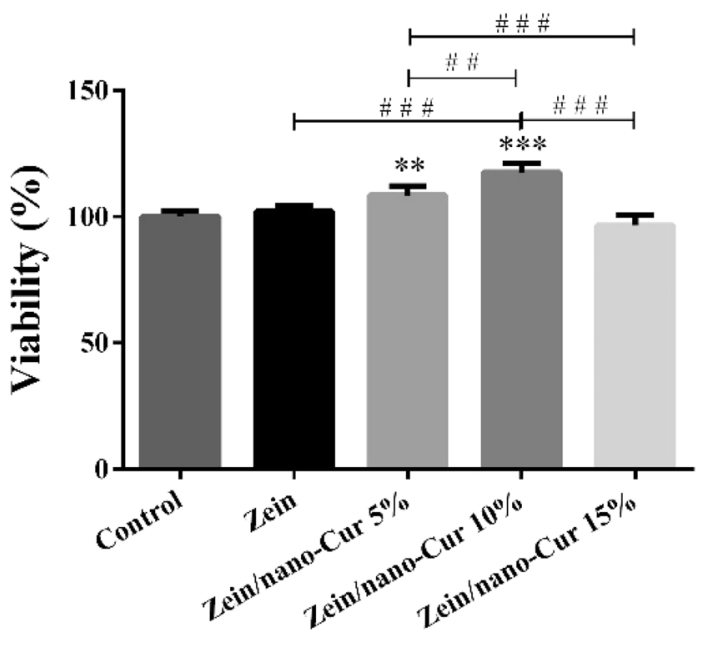

D) $\mathbf{L 9 2 9}$

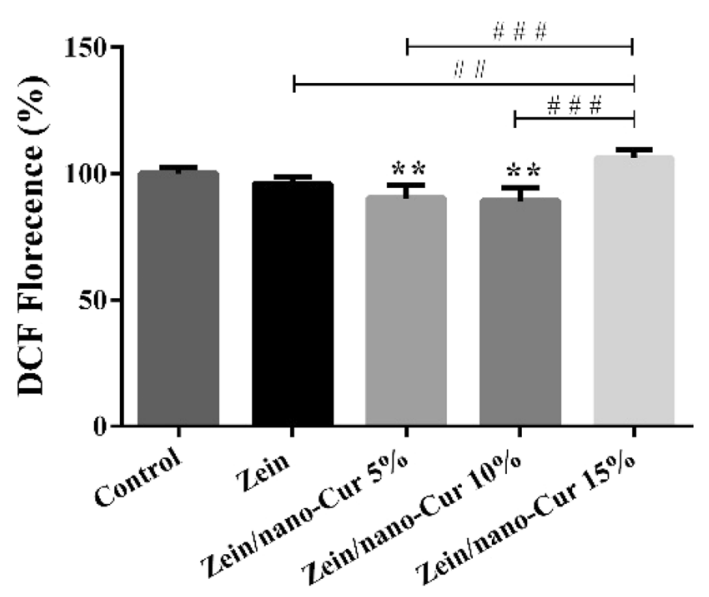

Figure 6. Viability (A, B), and oxidative stress (C, D) diagram of zein and zein/Nano-CUR 5\%, 10\%, and $15 \%$ NFs on HDF and L929 cell lines; \# indicates significance between groups and * indicates significance of every group with zein group $\left({ }^{\# / *} p<0.05,{ }^{\# 1^{* *}} p<0.01\right.$, and $\left.{ }^{\# \# \|^{* * *}} p<0.001\right)$.

$32.23 \pm 2.12 \%, 35.64 \pm 3.14 \%$, and $37.34 \pm 2.15 \%$, respectively. According to the statistical analysis, the wateruptakes of zein/Nano-CUR $10 \%$ and $15 \%$ have been significantly higher than that of the zein NFs.

Cytotoxicity test. We have evaluated the process of cell proliferation on zein and zein/Nano-CUR 5\%, 10\%, and $15 \%$ NFs through the usage of colorimetric MTT assay (Fig. 6A-B). In comparison to the case of control group, zein/Nano-CUR $5 \%$ and $10 \%$ mats have exhibited a significant increase in proliferation throughout the L929 and HDF cell lines.

Intracellular ROS. The intracellular free radicals of zein and zein/Nano-CUR 5\%, 10\%, and 15\% NFs have been assessed by the application of DCFH-DA colorimetric assay (Fig. 6C-D) and according to the gathered results, zein/Nano-CUR $10 \%$ has been capable of significantly reducing the existing free radicals in HDF cell media when compared to the control group. Also in comparison to the control group, the number of free radicals in L929 cell media has been significantly reduced by zein/Nano-CUR 5\% and 10\%.

Antibacterial study. The antibacterial properties of zein and zein/Nano-CUR NFs have been determined by the means of agar diffusion method (Fig. 7A-C). The antibiotic discs vancomycin, ciprofloxacin, and gentamicin have exhibited a $16 \mathrm{~mm}, 30 \mathrm{~mm}$, and $20 \mathrm{~mm}$ growth of inhibition zones, respectively, whereas zein and zein/Nano-CUR NFs had not displayed any growth of inhibition zone on Escherichia coli, Pseudomonas aeruginosa, and Staphylococcus aureus. 

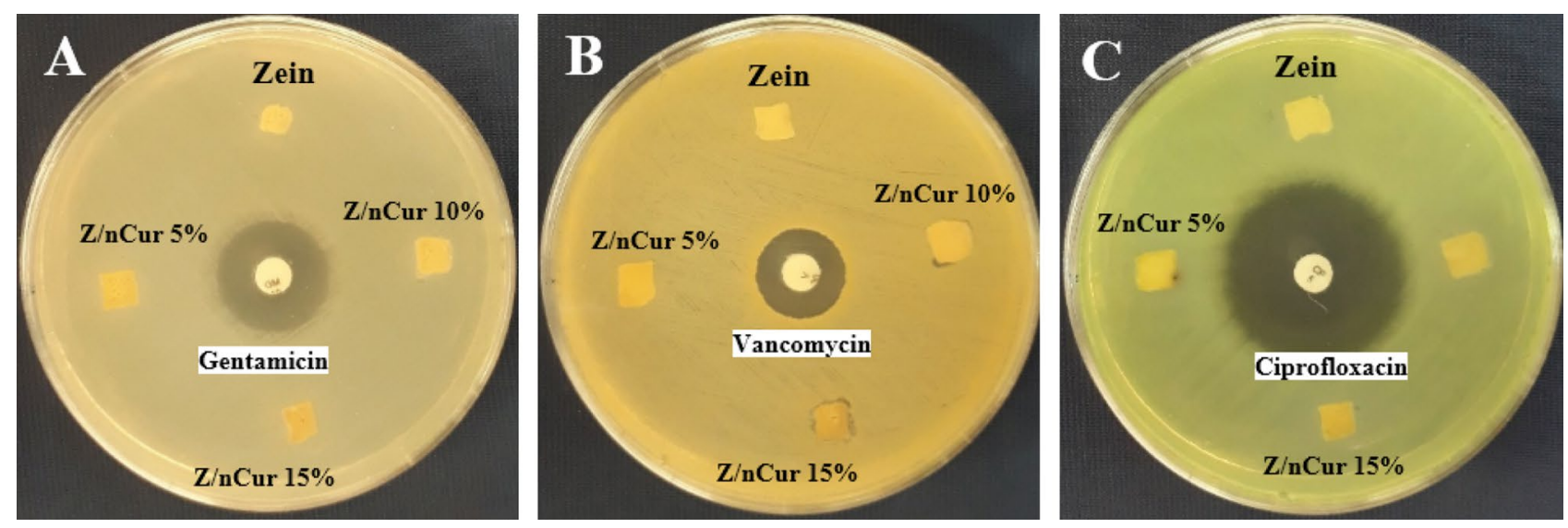

Figure 7. Evaluation of antibacterial properties of zein and zein/Nano-CUR NFs. (A) Escherichia coli, (B) Staphylococcus aureus, (C) Pseudomonas aeruginosa.

\section{Discussion}

There are several studies about the fabrication of curcumin in different polymers through the employment of electrospinning techniques and relatively the aim of this study has been set to demonstrate the possibility of developing the oil-in-water (O/W) nano-emulsion of curcumin (Nano-CUR), which would be loaded with zein NFs, and also evaluate their in vitro activity. Although curcumin is a hydrophobic polyphenol molecule with remarkable biological properties, yet due to containing a very low water solubility, many studies have attempted to increase its solubility and control its release for exerting its unique features in medical applications $\mathrm{s}^{70,71}$. In this work, we have utilized the O/W nano-emulsion of curcumin (Nano-CUR) while assuming that this alteration would be able to increase the chemical stability and solubility of curcumin and turn the resultant into a more effective substance in biological environment due to the small size and extended ratio of surface to volume.

The first aspect of NF studies has been performing investigations on the effects of electrospinning parameters on the NF morphology and diameter. According to our observations, NFs become significantly thinner and more uniformed as the portion of Nano-CUR is increased from 5 to $15 \%$. The obtained results has also indicated that the volume of porosity increases as the amount of Nano-CUR is enlarged, which could have been caused by the induced reduction in the diameter of nanofibers, as well as the improved uniformity of fibers and increased thickness of nanofibers. Subsequent to arranging similar conditions and evaluating the effective factors, the reduction of viscosity and extending spinnability have been the only parameters that had been observed to be capable of reducing the diameter and increasing the thickness. In comparison to the oily phase, oil/water emulsion Nano-CUR can reduce the viscosity of final solution through the addition of five times more water. The gathered outcomes have indicated that the viscosity of final zein solution has been reduced from 0.534 Pa.s to $0.267 \mathrm{~Pa} . \mathrm{s}$ in the final zein/Nano-CUR 15\% solution, suggesting that the viscosity of all the involved samples had been within the range of producing bead-free nanofibers. Meanwhile, our pilot attempt to produce zein/ Nano-CUR 20\% has led to the production of high beaded nanofibers, which is not mentioned in the results of this study. To the extent of our knowledge, surface tension is the predominant factor in a very low viscosity situation, while the spraying of polymer solution can lead to the formation of beaded nanofibers ${ }^{72}$. Furthermore, numerous studies have reported that increasing and reducing viscosity results in expanding and decreasing the diameter of nanofibers, respectively ${ }^{1,73}$. Having a higher evaporation rate of acetic acid than water could be considered as another reason behind the occurrence of an increase in the diameter of nanofibers as the volume of Nano-CUR is reduced, since this alteration can cause an increase in the viscosity of polymer solution at the tip of the needle and during the traveling of nanofibers towards being deposited on the collector ${ }^{74}$.

Next to the even distribution of Nano-CUR throughout the nanofibers, we have confirmed their lack of accumulation by observing the uniform fluorescent signals. Various studies have reported the fluorescent properties of curcumin ${ }^{75,76}$, which can be also detected by the usage of UV illumination or confocal laser scanning microscope $(\mathrm{CLSM})^{68}$. Furthermore, our study has displayed the capability of oil/water Nano-CUR in exhibiting the fluorescent signals in zein nanofibers, which is indicative of their high compatibility with zein polymer, the formation of a homogeneous solution even in the course of spinning, and confirming the stability of zein/ Nano-CUR homogenous solution during the electrospinning process.

There has not been any notable differences between the zein and zein/Nano-CUR spectra in the FTIR spectra, which may be due to the compatibly entrapment of Nano-CUR into zein NFs. The lack of observing any new peaks in the zein/Nano-CUR NFs has indicated the existence of a simple combination that does not contain any chemical bonds.

We have evaluated the factors of Young's modulus, elongation percentage at break point, and tensile strength for assessing the mechanical parameters of zein and zein/Nano-CUR NFs and according to the obtained outcomes, they can be improved by increasing the amount of Nano-CUR. As it has been previously stated, the diameter of NFs faces a reduction through the addition of Nano-CUR to zein scaffold; therefore, it seems to be quite logical to assume that causing a reduction in the average diameter of NFs and extending the number of nanofibers per unit area from zein/Nano-CUR 5\% to $15 \%$ would increase the tensile strength and young's 
modulus of NFs. Another reason behind the greater strength of zein NF with increased volume of Nano-CUR could be the occurrence of an increase in the thickness and uniformity of nanofibers.

Nano-CUR has been observed to be well dispersed in zein solution upon the addition of oil/water NanoCUR, which is probably due to the good mixture of water and acetic acid. Being in accordance with the SEM and fluorescent microscope images, the appearance of Nano-CURs can not be observed on the surface of nanofibers since they had been pushed towards the core of these substances. Apparently, as the polymer jet begins to move towards the collector, the acetic acid-soluble zein polymer is pulled further outwards due to the higher evaporation rate of acetic acid, while the aqueous phase that contains Nano-CUR shows a tendency to further move inwards. The in vitro release study of zein and zein/Nano-CUR NFs has displayed the occurrence of two phases throughout the releasing process of Nano-CUR from NFs, which had initially involved a burst release phase and a sustained release phase in the following. The burst release of Nano-CUR from the scaffold is caused as they move closer towards the surface of nanofibers. However, the impregnated Nano-CURs that exist deeper inside the polymer scaffold require more time to be released, which results in the creation of plateau phase. According to the obtained results, increasing the in vitro cumulative release and reducing EE\% is aligned with the occurrence of an increase in the portion of Nano-CUR, which can be legitimized along with the decreasing diameter of NF. Causing a reduction in the NF diameter can lead to an increase in the NF surface ratio and leave less available space inside the NF for the encapsulation of Nano-CUR; therefore, it can be assumed that a higher release is inevitable throughout the NFs that contain a smaller diameter. In conformity with the observations, there has been a higher amount of burst release in the case of zein/Nano-CUR $15 \%$, when compared to the results of $10 \%$ and $5 \%$ NFs, which could have been related to the narrower diameter of NFs. It has been reported by previous studies that thinner NFs had been able to display a faster in vitro release due to containing a larger surface area and wider contact with the outside environment ${ }^{20}$. In addition, the encapsulation of Nano-CUR in zein NFs has been more than $90 \%$ in our work, which proves the applicability of electrospinning technique for this particular purpose. Considering how the continuous release of a drug can be very beneficial, the case of chemotherapy drugs can be taken as an example since the effective concentration of this drug in the blood is preserved between the lowest effective levels and the maximum tolerable level during long periods ${ }^{46,77,78}$.

Results have indicated that the WVTR of zein NFs had been significantly higher than the scaffold of zein/ Nano-CUR $10 \%$ and $15 \%$. As it is known, controlling the occurrence of water vapor exchange through the nanofiber scaffolds is necessary for providing the proper repair of wounds, since high WVTR can cause rapid wound dehydration and result in leaving scars ${ }^{79}$. A low WVTR can cause the accumulation of exudates, result in delaying the process of wound healing, and increase the possibility of infection ${ }^{80}$. In regards to the performed evaluation of WVTR for burn wounds, Queen et al. have suggested that wound dressings which contain WVTR in the range of 2000-2500 g.m $\mathrm{m}^{-2}$ could provide a sufficient amount of moisture at the site of wound and avoid the inducement of dehydration ${ }^{12}$. Therefore, it can be assumed that zein/Nano-CUR $10 \%$ and $15 \%$ can facilitate a sufficient amount of moisture at the site of wound when being compared to zein and zein/Nano-CUR 5\%.

Results have indicated that the water-uptake capacity had been significantly increased by enlarging the volume of Nano-CUR, which could have been caused by the hydrophilicity of Nano-CUR. The essential role of water uptake capacity for absorbing extra exudates is quite evident since it can increase the risk of infection and maceration ${ }^{79}$. A high water absorption capacity can effectively increase the absorption of exudates from the wound bed and improve the nutritional perfusion, while water retention is useful in maintaining the moisture of wound bed, which results in increasing the migration of epidermal cells and re-epithelialization ${ }^{81}$. The observed increase in the WVTR and water uptake percentage could have been either caused by the extended hydrophilicity of zein nanofibers, which had occurred after the addition of Nano-CUR (Tween 80 is a hydrophilic nonionic surfactant $)^{82,83}$, or the higher porosity of zein at higher Nano-CUR percentages.

Various studies have investigated the effects of different types of nanofibers on the healing process of skin wounds and according to their date, a better cell attachment and proliferation can be provided by the application of nanofibers that had been synthesized through the electrospinning technique due to containing a high surfaceto-volume ratio. The results of these investigations have claimed that nanofibers in the range of 50-500 $\mathrm{nm}$ are similar in many aspects to the extracellular matrix and consequently, they can create an optimal environment for both fibroblasts' proliferation and matrix deposition ${ }^{84}$. In order to perform the biological evaluation of synthesized Nano-CUR, we have carried out the in vitro cytotoxicity and intracellular stress oxidative assessments. The obtained results have indicated that next to the lack of causing any cytotoxicity effect on HDF and L929 cell lines, zein/Nano-CUR 5\% and 10\% NFs have also increased the status of proliferation. According to a comparison between the cytotoxicity and stress oxidative results, the proliferation effect of Nano-CUR can be related to the inducement of a decrease in the oxidative stress. Compared to the case of zein/Nano-CUR $15 \%$ nanofibers, the increase in proliferation of zein/Nano-CUR 5 and $10 \%$ nanofibers may be the resultant of the existing difference in the release rate and volume of Nano-CUR, which is probably more compatible with the proliferation and removal of free radicals in zein/Nano-CUR 5 and 10\% nanofibers. It can be also suggested through this observation that the chemical structure of Nano-CUR in zein NFs is retained after the electrospinning process, while exhibiting cell proliferation and antioxidant properties in the presence of fibroblast cells. Our previous study on the antioxidant activity of emulsion components, oily phase, surfactants, and Nano-CUR has indicated that the antioxidant activity had been related to Nano-CUR. In addition, the effect of Nano-CUR cytotoxicity has not exhibited the inducement of any significant toxicity on Neuro2A cells up to the dosage of around $16 \mu \mathrm{g} / \mathrm{ml}^{49}$.

The antibacterial effect of nanofibers has not displayed any growth of inhibition zone throughout the cases of Escherichia coli, Pseudomonas aeruginosa, and Staphylococcus aureus. There are several studies that have reported the probable antibacterial effects of curcumin and its nano structures ${ }^{85-88}$, however, further investigations are required to figure out why there has not been any detected antibacterial impacts from the zein/NanoCUR NFs in this study. One of the possible reasons that should be evaluated is the low amount of available Nano-CUR in nanofibers, which can be tested by increasing its content, while another assumption that may be 
effective is our selected method for investigating the antibacterial effect. Throughout the agar diffusion method, Nano-CURs are limited in being released from all the involved surfaces of nanofibers in order to come into contact with bacteria, which can be considered as one of the disadvantages of this procedure. This factor can be evaluated through the utilization of antimicrobial gradient or dilution methods ${ }^{89}$ since they can provide a better release of Nano-CURs and have them entirely encountered with bacteria.

\section{Conclusion}

In this study, we have prepared zein containing Nano-CUR NFs at three volumes of 5\%, 10\%, and 15\% (v/v) through the employment of electrospinning technique. Thereafter, the physicochemical properties of synthesized NFs, including viscosity, FESEM, FTIR, tensile strength, pore size, encapsulation efficiency, release profile, WVTR, and water-uptake capacity, have been investigated along with evaluating the biological properties such as cytotoxicity and antioxidant assessments on human fibroblast (HDF) and mouse (L929) cell lines. Our work has resulted in obtaining suitable electrospinning parameters that led to the fabrication of uniformed and nonbeaded nanofibers. Moreover, we have observed a significant reduction in the mean diameter of zein NFs, as well as the occurrence of an increase in the mechanical properties, by extending the percentage of Nano-CUR. The higher percentage of Nano-CUR loaded NFs have also exhibited higher in vitro release and lower encapsulation efficiency than the other groups of Nano-CUR loaded NFs. According to the results of viability and antioxidant studies, zein/Nano-CUR 10\% NFs are capable of providing the best conditions for cell proliferation. Overall, we have suggested in this study that Nano-CUR can be successfully woven in zein NFs and maintain their biological properties.

\section{Material and methods}

Materials. The following materials have been purchased in analytical grade and used without further purification: Zein (Sigma-Aldrich, Spain), Curcumin (>99\%, Merck, Germany; MW=368.38 g/mol), Tween $80\left(\mathrm{C}_{64} \mathrm{H}_{124} \mathrm{O}_{26}\right.$, Merck, Germany), Oil extract of black pepper (Golsorkh-Exir Co., Iran), Glacial acetic acid (>99.7\%, Dr. Mojallali, Iran), Dimethylthiazol-diphenyl tetrazolium bromide (MTT) (>98\%, Sigma-Aldrich, USA), Dichloro-dihydro-fluorescein diacetate (DCFH-DA) (>98\%, Sigma-Aldrich, USA), Dimethyl-sulfoxide (DMSO) (>99.9\%, Sigma- Aldrich, USA), Dulbecco's Modified Eagle Medium (DMEM) (Invitrogen Co, USA), and Ethanol (>96\%, Dr. Mojallali, Iran). Deionized water (DW) has been applied in all of the involved experiments. L929 cell line (NCBI Code: C161) was purchased from Pasteur Institute of Iran and HDF cell line was isolated from human neonatal foreskin samples by Keira et al. protocol ${ }^{90}$. The protocol approved by Ethics Committee of Mashhad University of Medical Sciences (code number: 940891), through obtaining an informed consent from neonatal parents and all the investigations were conducted in accordance with the Declaration of Helsinki.

Nano-CUR synthesize. The required Nano-CUR has been prepared in accordance with the previously reported method ${ }^{49}$. Briefly, the preparation of Nano-CUR through the means of oil in water $(\mathrm{O} / \mathrm{W})$ emulsion has been carried out under three phases. To prepare the oily phase, the mixture of oil black pepper (BP) and surfactant (Tween 80$)(1 \mathrm{~g}: 9 \mathrm{~g}(\mathrm{w} / \mathrm{w}))$ has been stirred for $15 \mathrm{~min}$ and then, $100 \mathrm{mg}$ of curcumin has been stirred in the oily phase while being under magnetic stirring at $500 \mathrm{rpm}$ for $2 \mathrm{~h}$ at the specified temperature. The resulting mixture has been homogenized in an ultrasonic bath (DT510, Bandelin, $35 \mathrm{kHz}$, Germany) for $1 \mathrm{~h}$ to remove the existing air bubbles and obtain a homogeneous yellow solution. The final emulsion had been achieved by appending the DW to oily phase $(5: 1 \mathrm{v} / \mathrm{v})$ and have it stirred at $500 \mathrm{rpm}$ for another $30 \mathrm{~min}$, which had been determined to be $1000 \mu \mathrm{g} / \mathrm{ml}$ at the end of procedure.

Preparation of zein and zein/Nano-CUR solution. Zein $(25 \% \mathrm{w} / \mathrm{w})$ has been dissolved in acid acetic glacial and stirred for the duration of $12 \mathrm{~h}$ to homogenize the solution. To prepare the zein/Nano-CUR solution, $5 \%, 10 \%$, and $15 \%(\mathrm{v} / \mathrm{v})$ of the synthesized Nano-CUR has been added into the zein solution to retain the final concentration of $25 \%$. Thereafter, the mixture has been stirred for $12 \mathrm{~h}$ through the usage of magnetic stirrer. The prepared zein and zein/Nano-CUR solutions have been left aside for a day at room temperature until the bubbles had been removed.

Polymer viscosity measurement. The dynamic viscosity of zein and zein/Nano-CUR NFs has been measured by the means of rotating viscometer (Brookfield Co., DV-III Ultra, USA) at room temperature, which had involved the usage of spindle type CC25 at a constant shear rate. The viscosity of zein and zein/Nano-CUR solutions has been reported at the shear rate of $100 \mathrm{~s}^{-1}$.

Electrospinning process. As it is presented in Fig. 8, Zein and zein/Nano-CUR NFs have been fabricated through the utilization of an electrospinning device that had been equipped with a high voltage power supplier $(0-30 \mathrm{kV})$ (Fnm Co., ES1000, Iran). The available $5 \mathrm{ml}$ plastic syringes have been filled with the solutions of zein and zein/Nano-CUR while being connected to a 16-gauge stainless steel needle. The solutions have been fed into the needle by a syringe pump at $2.5 \mathrm{ml} / \mathrm{h}$, which had been attached to the positive electrode while applying the electrospinning voltage of $25 \mathrm{kV}$. The created positively charged polymer at the Taylor cone has been observed to move from the tip of needle towards the collector surface that had been covered by aluminum foil. The traveled distance between the needle and collector has been measured to be $150 \mathrm{~mm}$. It should be noted that entire procedure of electrospinning has been carried out at room temperature. 


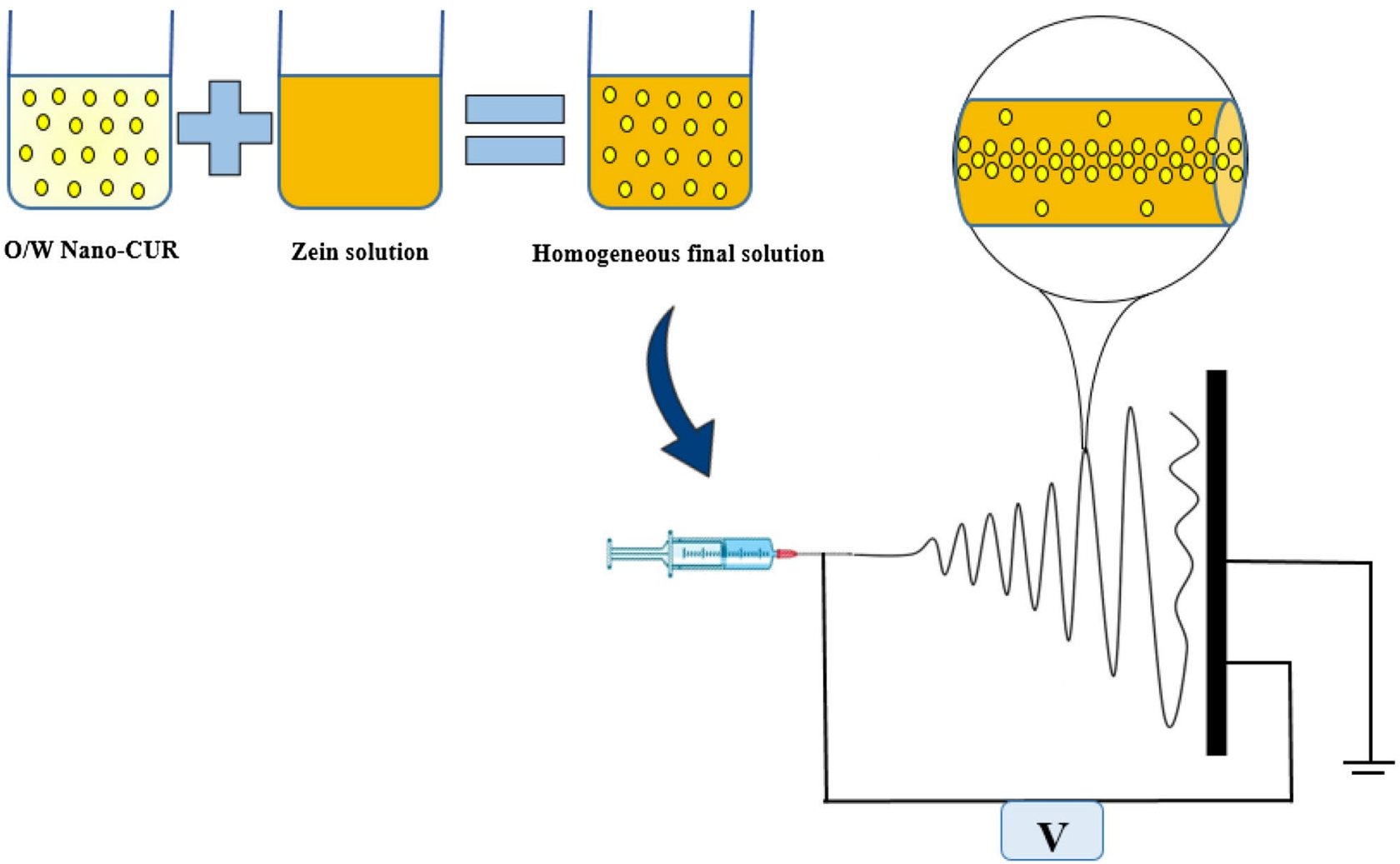

Figure 8. Schematic illustration of the synthesis of zein/Nano-CUR nanofibers.

Surface morphology. We have analyzed the surface morphology of zein and zein/Nano-CUR NFs through the employment of Field Emission-Scanning Electron Microscopy (FESEM) (MIRA3, TESCAN Co., Czech). The images have been taken at the accelerating voltage of $20 \mathrm{kV}$ subsequent to performing the gold coating process (Q150R ES, Quorum Technologies, UK). We have measured the diameters of NF by the usage of Image-J software (https://imagej.net/Fiji) along with the sample size of $100 \mathrm{NF}$. In addition, the fibers that had contained fluorescent properties have been identified under the Olympus fluorescent microscope (IX53, Japan), which had been equipped with a digital camera (Olympus DP73, Japan).

Mechanical properties. The mechanical properties of zein and zein/Nano-CUR NFs mats have been determined through the utilization of a universal testing machine (Hounsfield, England) that had contained $10 \mathrm{~N}$ of load capacity and $5 \mathrm{~mm} / \mathrm{min}$ of tensile speed. The NF mats have been cut into $4 \times 1 \mathrm{~cm}$ sections and a minimum of five measurements had been performed for each specimen. In addition, we have also measured their thickness by the means of digital caliper, which had been carried out in five randomized areas of NFs.

Fourier transform-infrared spectroscopy (FT-IR). We have carried out the Fourier transform-infrared spectroscopy (Thermo Nicolet, AVATAR 370, FTIR) analysis of zein-Nano-CUR NFs for the purpose of studying the existing interaction among constituents. The obtained spectra have been displayed at the wavenumber range of $4000-400 \mathrm{~cm}^{-1}$.

Porosity measurements. The porosity of zein and zein/Nano-CUR NFs has been measured through the employment of a micro-volumetric modification of liquid displacement method that had been developed by Moradi et al. ${ }^{91}$. Briefly, subsequent to the immersion of scaffold in a glass pipette (V1) and after having it removed (V2), the induced changes in hexane surface had been recorded by a digital camera, which had been later on evaluated through the usage of ImageJ software (https://imagej.net/Fiji). The porosity measurements have been performed for fifteen times and the results had been reported as mean \pm SD. The pore volume (\%) can be calculated through the following equation (Eq. 1):

$$
\text { Total pore volume }=\frac{V 2}{V 1+V 2} \times 100
$$

In vitro drug release study. The in vitro cumulative release study of Nano-CUR from zein NFs has been carried out in a specified immersion period for the duration of 20 days. As the next step, the membranes have been cut into the diameters of $2 \times 1 \mathrm{~cm}^{2}$, accurately weighed, immersed in $4 \mathrm{ml}$ of phosphate buffer saline (PBS, 
$\mathrm{pH}=7.4$ ), and incubated in the shaker incubator (JTS 40, Iran) at $37^{\circ} \mathrm{C}$. At the given time intervals, the samples have been transferred to a new medium and analyzed by the utilization of UV-spectrophotometry (CE9500, CECIL, UK) at $425 \mathrm{~nm}$. We have performed all of the involved release studies in triplicate and had the obtained results reported as mean $\pm \mathrm{SD}$.

Determination of encapsulation efficiency (\%). Encapsulation efficiency is known as the percentage of a drug that has been successfully entrapped after the formulation process. The encapsulation efficiency percentage of Nano-CUR in NFs has been calculated by analyzing the amount of existing Nano-CUR in the supernatant of $2 \times 1 \mathrm{~cm}^{2}$, having it accurately weighed, and performing the centrifugation of NFs at $15,000 \mathrm{~g}$ for $15 \mathrm{~min}$. Furthermore, the optical absorbance has been measured at 425 and $630 \mathrm{~nm}$ (as reference wavelength) by the usage of UV-visible spectrophotometer (CE9500, CECIL, UK), while the amount of Nano-CUR in supernatant has been determined through the standard curve of designated concentrations. Being the remaining amount of Nano-CUR in NF, the encapsulation efficiency (\%) can be calculated by applying the following equation (Eq. 2). We have performed all of the EE\% studies in triplicate and had the obtained results reported as mean $\pm \mathrm{SD}$.

$$
E E(\%)=\frac{\text { EncapsulatedNano }- \text { CUR }}{\text { TotalNano }- \text { CUR }} \times 100(2)
$$

Water-uptake capacity. To calculate the absorption capacity of wound exudates by nanofiber scaffolds, the water-uptake capacity has been calculated through the following Eq. ${ }^{92,93}$.

$$
\text { Water - uptakecapacity }(\%)=\frac{W w-W d}{W d} \times 100
$$

In which $\mathrm{W}_{\mathrm{d}}$ stands as the primary sample weight $(1 \times 1 \mathrm{~cm})$ and $\mathrm{W}_{\mathrm{w}}$ represents the sample weight subsequent to being immersed in PBS for $24 \mathrm{~h}$ at room temperature. We have repeated the process of water- uptake capacity for three times in regards to each sample and had the results reported as mean \pm standard deviation.

Water vapor transmission rate (WVTR). Nanofiber scaffolds have been exposed to an open round container with a diameter of $1.2 \mathrm{~cm}$ for the purpose of calculating the amount of Water vapor transmission rate. In the following, we have filled the container with $5 \mathrm{ml}$ of sterilized water, which is about one quarter of the container's height, and had the vessel placed in an oven at $35{ }^{\circ} \mathrm{C}$ with the humidity of $35 \%$ for $24 \mathrm{~h}$. Lastly, the WVTR has been calculated by the usage of the following Eq. ${ }^{12,92}$.

$$
W V T R=\frac{\Delta W}{A}
$$

where $\Delta \mathrm{W}$ represents the change of water weight $(\mathrm{g})$ and $\mathrm{A}$ is the open surface area of the container $\left(1.13 \times 10^{-4}\right.$ $\mathrm{m}^{2}$ ). A round container without nanofiber scaffolds has been considered as a control group. The amount of WVTR has been calculated for three times in regards to each sample and the results had been reported as mean \pm standard deviation.

In vitro cytotoxicity study. We have evaluated the cytotoxicity of zein and zein/Nano-CUR NFs by performing the MTT assay on human dermal fibroblast cell line (HDF) and mouse fibroblast cell line (L929) ${ }^{34,35,94}$. The HDF and L929 cells have been cultured in high glucose cell culture medium (DMEM) while containing $10 \%$ FBS and $0.5 \%$ penicillin/streptomycin, which had been then incubated at $37^{\circ} \mathrm{C}$ with $10 \% \mathrm{CO}_{2}$ and $90 \%$ humidity. The UV sterilized, $8 \mathrm{~mm}$-circular NFs have been positioned at the bottom of 96 -well plate and pre-wetted with culture medium at $37^{\circ} \mathrm{C}$. Subsequent to reaching a confluency of $70 \%$, the cells have been harvested with $0.25 \%$ trypsin and 5000 cells, in passages of 3 or 4 , which had been afterwards seeded in each well of the NFs membranes. As the next step, we have incubated the plate at $37^{\circ} \mathrm{C}$ with $10 \% \mathrm{CO}_{2}$ and $90 \%$ humidity for allowing the cells to proliferate on the membrane for $48 \mathrm{~h}$. Meanwhile, Non-seeded membranes have been considered as the negative control. Subsequent to $48 \mathrm{~h}, 10 \mu \mathrm{l}$ of MTT $(5 \mathrm{mg} / \mathrm{ml})$ in PBS has been added to each well and once $4 \mathrm{~h}$ of incubation at $37^{\circ} \mathrm{C}$ had passed, the wells have been emptied from culture media and replaced with $100 \mu \mathrm{l}$ of DMSO to dissolve the existing formazan crystals. To conclude the procedure, the optical absorbance has been measured at 540 and $630 \mathrm{~nm}$ (as reference wavelength) through the usage of a microplate reader (Epoch, BioTeck, USA). All of the involved experiments have been repeated for five times.

In vitro Antioxidant activity. The oxidative stress of zein/Nano-CUR NFs has been evaluated by utilizing a DCFH-DA colorimetric quantitative method ${ }^{95,96}$. We have seeded the HDF and L929 cells on the membranes that had been positioned at the bottom of each well, which had been thoroughly described in the section of cytotoxicity method. The prepared 96 -well plate has been incubated at $37^{\circ} \mathrm{C}$ with $10 \%$ of $\mathrm{CO}_{2}$ and $90 \%$ of humidity, while the non-seeded membranes have been considered as the negative control. Subsequent to $48 \mathrm{~h}$, the supernatant of cells has been evacuated and $10 \mu \mathrm{L}$ of freshly prepared CFH-DA dye $(10 \mu \mathrm{M})$ had been added to the FBS free medium. Once $40 \mathrm{~min}$ of incubation at $37^{\circ} \mathrm{C}$ had passed, the fluorescence intensity has been recorded by the employment of a fluorescence plate reader (VICTOR X5, PerkinElmer) in the excitation of $485 \mathrm{~nm}$ and the emission of $630 \mathrm{~nm}$. We have repeated every experiment for five times. 
Agar diffusion antibacterial test. Three bacterial strains of Escherichia coli, Pseudomonas aeruginosa (Gram-negative), and Staphylococcus aureus (Gram-positive), which are known as the most common hospital skin wound bacteria, have been exerted to evaluate the antibacterial properties of nanofiber scaffolds through the means of agar diffusion method. In this regard, we have carefully spread $100 \mu$ lof bacterial solution $\left(10^{6} \mathrm{CFU} /\right.$ $\mathrm{ml})$ on the mueller hinton agar plate and had zein and zein/Nano-CUR NFs $\left(1 \mathrm{~cm}^{2}\right)$ placed on the plate surface. Vancomycin $(30 \mu \mathrm{g})$, gentamicin $(10 \mu \mathrm{g})$, and ciprofloxacin $(10 \mu \mathrm{g})$ antibiotic discs have been applied as the positive control for Staphylococcus aureus, Escherichia coli, and Pseudomonas aeruginosa, respectively. The inhibition zone has been examined after $24 \mathrm{~h}$ of incubation at $37^{\circ} \mathrm{C}$.

Statistical analysis. Statistical analysis was evaluted by two-way analysis of variance (ANOVA) and Tukey post-hoc test. A p-value 0.05 was considered statistically significant. GraphPad Prism 6 software was applied to analyze the data.

Received: 22 June 2020; Accepted: 21 September 2020

Published online: 21 January 2021

\section{References}

1. Ramakrishna, S. An introduction to electrospinning and nanofibers (World Scientific, Singapore, 2005).

2. Zhang, Y., Lim, C. T., Ramakrishna, S. \& Huang, Z. M. Recent development of polymer nanofibers for biomedical and biotechnological applications. J. Mater. Sci. Mater. Med. 16, 933-946 (2005).

3. 3Song, B., Wu, C. \& Chang, J. Controllable delivery of hydrophilic and hydrophobic drugs from electrospun poly (lactic-co-glycolic acid)/mesoporous silica nanoparticles composite mats. J. Biomed. Mater. Res. B Appl. Biomater.100, 2178-2186 (2012).

4. Xu, J., Jiao, Y., Shao, X. \& Zhou, C. Controlled dual release of hydrophobic and hydrophilic drugs from electrospun poly (l-lactic acid) fiber mats loaded with chitosan microspheres. Mater. Lett. 65, 2800-2803 (2011).

5. Hassan, M. A., Yeom, B. Y., Wilkie, A., Pourdeyhimi, B. \& Khan, S. A. Fabrication of nanofiber meltblown membranes and their filtration properties. J. Membr. Sci. 427, 336-344 (2013).

6. Ma, P. X. \& Zhang, R. Synthetic nano-scale fibrous extracellular matrix. J. Biomed. Mater. Res. 46, 60-72 (1999).

7. Martin, C. R. Membrane-based synthesis of nanomaterials. Chem. Mater. 8, 1739-1746 (1996).

8. Heunis, T. \& Dicks, L. Nanofibers offer alternative ways to the treatment of skin infections. Biomed Res. Int. 2010, 1-10 (2010).

9. 9Jain, K. K. in Drug Deliv. Syst. 1-50 (Springer, Berlin, 2008).

10. 10Motealleh, B. et al. Morphology, drug release, antibacterial, cell proliferation, and histology studies of chamomile-loaded wound dressing mats based on electrospun nanofibrous poly ( $\varepsilon$-caprolactone)/polystyrene blends. J. Biomed. Mater. Res. B Appl. Biomater.102, 977-987 (2014).

11. Jones, V., Grey, J. E. \& Harding, K. G. Wound dressings. The BMJ 332, 777-780 (2006).

12. Queen, D., Gaylor, J., Evans, J., Courtney, J. \& Reid, W. The preclinical evaluation of the water vapour transmission rate through burn wound dressings. Biomaterials 8, 367-371 (1987).

13. Wang, A., Xu, C., Zhang, C., Gan, Y. \& Wang, B. Experimental investigation of the properties of electrospun nanofibers for potential medical application. J. Nanomater. 2015, 1-8 (2015).

14. Reddy, N. \& Yang, Y. Potential of plant proteins for medical applications. Trends Biotechnol. 29, 490-498 (2011).

15. Paliwal, R. \& Palakurthi, S. Zein in controlled drug delivery and tissue engineering. J. Control. Release 189, 108-122 (2014).

16. Kong, B. \& Xiong, Y. L. Antioxidant activity of zein hydrolysates in a liposome system and the possible mode of action. J. Agric. Food Chem. 54, 6059-6068 (2006).

17. Shukla, R. \& Cheryan, M. Zein: the industrial protein from corn. Ind. Crops Prod. 13, 171-192 (2001).

18. Miyoshi, T., Toyohara, K. \& Minematsu, H. Preparation of ultrafine fibrous zein membranes via electrospinning. Polym. Int. 54, $1187-1190$ (2005).

19. Torres-Giner, S., Gimenez, E. \& Lagarón, J. M. Characterization of the morphology and thermal properties of zein prolamine nanostructures obtained by electrospinning. Food Hydrocoll. 22, 601-614 (2008).

20. Li, Y. \& Zhang, T. Targeting cancer stem cells by curcumin and clinical applications. Cancer Lett. 346, 197-205 (2014).

21. Eigner, D. \& Scholz, D. Ferula asa-foetida and Curcuma longa in traditional medical treatment and diet in Nepal. J. Ethnopharmacol. 67, 1-6 (1999).

22. Aggarwal, B. B., Yuan, W., Li, S. \& Gupta, S. C. Curcumin-free turmeric exhibits anti-inflammatory and anticancer activities: Identification of novel components of turmeric. Mol. Nutr. Food Res. 57, 1529-1542 (2013).

23. Chin, D., Huebbe, P., Pallauf, K. \& Rimbach, G. Neuroprotective properties of curcumin in Alzheimer's disease-merits and limitations. Curr. Med. Chem. 20, 3955-3985 (2013).

24. Rajasekhar Reddy, A. et al. A comprehensive review on sar of curcumin. Mini-Rev. Med. Chem. 13, 1769-1777 (2013).

25. Sun, M. et al. Advances in nanotechnology-based delivery systems for curcumin. Nanomedicine 7, 1085-1100 (2012).

26. Sharma, R., Gescher, A. \& Steward, W. Curcumin: the story so far. Eur. J. Cancer 41, 1955-1968 (2005).

27. Lao, C. D. et al. Dose escalation of a curcuminoid formulation. BMC Complement Altern. Med. 6, 1-4 (2006).

28. Aggarwal, B. B. \& Sung, B. Pharmacological basis for the role of curcumin in chronic diseases: an age-old spice with modern targets. Trends Pharmacol. Sci. 30, 85-94 (2009).

29. Anand, P., Kunnumakkara, A. B., Newman, R. A. \& Aggarwal, B. B. Bioavailability of curcumin: problems and promises. Mol. Pharm. 4, 807-818 (2007).

30. Yang, C. et al. Advances in clinical study of curcumin. Curr. Pharm. Des. 19, 1966-1973 (2013).

31. Bisht, S. \& Maitra, A. Systemic delivery of curcumin: 21 st century solutions for an ancient conundrum. Curr. Drug Discov. Technol. 6, 192-199 (2009).

32. Guo, G. et al. Preparation of curcumin loaded poly ( $\varepsilon$-caprolactone)-poly (ethylene glycol)-poly ( $\varepsilon$-caprolactone) nanofibers and their in vitro antitumor activity against Glioma 9L cells. Nanoscale 3, 3825-3832 (2011).

33. Bui, H. T., Chung, O. H., Cruz, J. D. \& Park, J. S. Fabrication and characterization of electrospun curcumin-loaded polycaprolactone-polyethylene glycol nanofibers for enhanced wound healing. Macromol. Res. 22, 1288-1296 (2014).

34. Fu, S. Z. et al. Acceleration of dermal wound healing by using electrospun curcumin-loaded poly ( $\varepsilon$-caprolactone)-poly (ethylene glycol)-poly ( $\varepsilon$-caprolactone) fibrous mats. J. Biomed. Mater. Res. B. 102, 533-542 (2014).

35. Merrell, J. G. et al. Curcumin-loaded poly ( $\varepsilon$-caprolactone) nanofibres: Diabetic wound dressing with anti-oxidant and antiinflammatory properties. Clin. Exp. Pharmacol. Physiol. 36, 1149-1156 (2009).

36. Ranjbar-Mohammadi, M. \& Bahrami, S. H. Electrospun curcumin loaded poly(ع-caprolactone)/gum tragacanth nanofibers for biomedical application. Int. J. Biol. Macromol. 84, 448-456 (2016).

37. Dhurai, B. et al. Electrospinning of curcumin loaded chitosan/poly (lactic acid) nanofilm and evaluation of its medicinal characteristics. Front. Mater. Sci. 7, 350-361 (2013). 
38. Mo, Y. et al. Controlled dual delivery of angiogenin and curcumin by electrospun nanofibers for skin regeneration. Tissue Eng. Part A 23, 597-608 (2017).

39. Mei, L. et al. Nanofibers for improving the wound repair process: The combination of a grafted chitosan and an antioxidant agent. Polym. Chem. 8, 1664-1671 (2017).

40. Perumal, G. et al. Synthesis and characterization of curcumin loaded PLA-Hyperbranched polyglycerol electrospun blend for wound dressing applications. Mater. Sci. Eng. C 76, 1196-1204 (2017).

41. Singaravelu, S., Ramanathan, G. \& Sivagnanam, U. T. Dual-layered 3D nanofibrous matrix incorporated with dual drugs and their synergetic effect on accelerating wound healing through growth factor regulation. Mater. Sci. Eng. C 76, 37-49 (2017).

42. Gandhimathi, C., Venugopal, J. R., Bhaarathy, V., Ramakrishna, S. \& Kumar, S. D. Biocomposite nanofibrous strategies for the controlled release of biomolecules for skin tissue regeneration. Int. J. Nanomedicine 9, 4709-4722 (2014).

43. Mutlu, G., Calamak, S., Ulubayram, K. \& Guven, E. Curcumin-loaded electrospun PHBV nanofibers as potential wound-dressing material. J. Drug Deliv. Sci. Technol. 43, 185-193 (2018).

44. Fereydouni, N. et al. Curcumin nanofibers for the purpose of wound healing. J. Cell. Physiol. 234, 5537-5554 (2019).

45. Bulboacă, A. E. et al. Liposomal curcumin is better than curcumin to alleviate complications in experimental diabetic mellitus. Molecules 24, 1-17 (2019).

46. Das, R. K., Kasoju, N. \& Bora, U. Encapsulation of curcumin in alginate-chitosan-pluronic composite nanoparticles for delivery to cancer cells. Nanomedicine 6, 153-160 (2010).

47. He, X. et al. Antidepressant effects of curcumin and HU-211 coencapsulated solid lipid nanoparticles against corticosteroneinduced cellular and animal models of major depression. Int. J. Nanomedicine 11, 4975-4990 (2016).

48. Nosrati, H., Danafar, H., Rezaeejam, H., Gholipour, N. \& Rahimi-Nasrabadi, M. Evaluation radioprotective effect of curcumin conjugated albumin nanoparticles. Bioorg. Chem. 100, 1-7 (2020).

49. Moghaddasi, F., Housaindokht, M. R., Darroudi, M., Bozorgmehr, M. R. \& Sadeghi, A. Synthesis of nano curcumin using black pepper oil by O/W nanoemulsion technique and investigation of their biological activities. LWT 92, 92-100 (2018).

50. Kim, S., Diab, R., Joubert, O., Canilho, N. \& Pasc, A. Core-shell microcapsules of solid lipid nanoparticles and mesoporous silica for enhanced oral delivery of curcumin. Colloids Surf. B Biointerfaces 140, 161-168 (2016).

51. Flora, G., Gupta, D. \& Tiwari, A. Nanocurcumin: a promising therapeutic advancement over native curcumin. Crit. Rev. Ther. Drug 30, 331-368 (2013).

52. Rachmawati, H., Yee, C. W. \& Rahma, A. Formulation of tablet containing curcumin nanoemulsion. Int. J. Pharm. Pharm. Sci. 6, $115-116(2014)$.

53. Pourmohamadian, H., Rahimi-Nasrabadi, M., Sheikhzadeh, G. A. \& Tabrizi, H. B. Preparation of SrTiO 3-microencapsulated palmitic acid by means of a sol-gel approach as thermal energy storage materials. J. Mater. Sci.: Mater Electron. 29, 794-800 (2018).

54. Pourmohamadian, H., Sheikhzadeh, G. A., Rahimi-Nasrabadi, M. \& Tabrizi, H. B. Fabrication and characterization of microencapsulated PA with $\mathrm{SiO} 2$ shell through sol-gel synthesis via sodium silicate precursor. J. Mater. Sci. Mater. Electron. 28, 9990-9997 (2017).

55. 55Sobhani-Nasab, A., Pourmohamadian, H., Rahimi-Nasrabadi, M., Sheikhzadeh, G. A. \& Tabrizi, H. B. Evaluation of the thermal properties of $\mathrm{SrCO} 3$-microencapsulated palmitic acid composites as thermal energy storage materials. J. Therm. Anal. Calorim., 1-8 (2019).

56. Pourmohamadian, H., Rahimi-Nasrabadi, M., Sobhani-Nasab, A., Sheikhzadeh, G. A. \& Tabrizi, H. B. Experimental study of the thermal properties of microencapsulated palmitic acid composites with $\mathrm{CuCO} 3$ shell as thermal energy storage materials. ChemistrySelect 4, 6501-6505 (2019).

57. Syed, I., Banerjee, P. \& Sarkar, P. Oil-in-water emulsions of geraniol and carvacrol improve the antibacterial activity of these compounds on raw goat meat surface during extended storage at $4{ }^{\circ} \mathrm{C}$. Food Control 107, 1-11 (2020).

58. Zhu, Q. et al. Review on the stability mechanism and application of water-in-oil emulsions encapsulating various additives. Compr. Rev. Food Sci. Food Saf. 18, 1660-1675 (2019).

59. Raviadaran, R., Chandran, D., Shin, L. H. \& Manickam, S. Optimization of palm oil in water nano-emulsion with curcumin using microfluidizer and response surface methodology. LWT 96, 58-65 (2018).

60. García-Moreno, P. J., Guadix, A., Guadix, E. M. \& Jacobsen, C. Physical and oxidative stability of fish oil-in-water emulsions stabilized with fish protein hydrolysates. Food Chem. 203, 124-135 (2016).

61. Ospina, M., Montaña-Oviedo, K., Díaz-Duque, Á, Toloza-Daza, H. \& Narváez-Cuenca, C.-E. Utilization of fruit pomace, overripe fruit, and bush pruning residues from Andes berry (Rubus glaucus Benth) as antioxidants in an oil in water emulsion. Food Chem. 281, 114-123 (2019).

62. Chaari, M., Theochari, I., Papadimitriou, V., Xenakis, A. \& Ammar, E. Encapsulation of carotenoids extracted from halophilic Archaea in oil-in-water (O/W) micro-and nano-emulsions. Colloids Surf. B Biointerfaces 161, 219-227 (2018).

63. Saani, S. M., Abdolalizadeh, J. \& Heris, S. Z. Ultrasonic/sonochemical synthesis and evaluation of nanostructured oil in water emulsions for topical delivery of protein drugs. Ultrason. Sonochem. 55, 86-95 (2019).

64. 64Brito, L., Chan, M., Geall, A., O'hagan, D. \& Singh, M. (Google Patents, 2017).

65. Jung, Y., Yang, H., Lee, I.-Y., Yong, T.-S. \& Lee, S. Core/sheath-structured composite nanofibers containing cinnamon oil: their antibacterial and antifungal properties and acaricidal effect against house dust mites. Polymers 12, 1-18 (2020).

66. Basar, A., Castro, S., Torres-Giner, S., Lagaron, J. \& Sasmazel, H. T. Novel poly ( $\varepsilon$-caprolactone)/gelatin wound dressings prepared by emulsion electrospinning with controlled release capacity of Ketoprofen anti-inflammatory drug. Mater. Sci. Eng. C 81, 459-468 (2017).

67. García-Moreno, P. J. et al. Encapsulation of fish oil in nanofibers by emulsion electrospinning: physical characterization and oxidative stability. J. Food Eng. 183, 39-49 (2016).

68. Brahatheeswaran, D. et al. Hybrid fluorescent curcumin loaded zein electrospun nanofibrous scaffold for biomedical applications. Biomed. Mater. 7, 1-16 (2012).

69. Khan, Y. et al. Low temperature synthesis of fluorescent ZnO nanoparticles. Appl. Surf. Sci. 257, 1756-1761 (2010).

70. Maheshwari, R. K., Singh, A. K., Gaddipati, J. \& Srimal, R. C. Multiple biological activities of curcumin: a short review. Life Sci. 78, 2081-2087 (2006).

71. Akbik, D., Ghadiri, M., Chrzanowski, W. \& Rohanizadeh, R. Curcumin as a wound healing agent. Life Sci. 116, 1-7 (2014).

72. Bhattarai, R. S., Bachu, R. D., Boddu, S. H. \& Bhaduri, S. Biomedical applications of electrospun nanofibers: drug and nanoparticle delivery. Pharmaceutics 11, 1-30 (2019).

73. 73Li, Z. \& Wang, C. in One-dimensional nanostructures 15-28 (Springer, 2013).

74. Horuz, T. I. \& Belibağli, K. B. Effects of solvent type and process parameters on electrospinnability of zein through orthogonal experimental design. Mater. Sci. 24, 10-17 (2018).

75. Mogharbel, B. F. et al. Fluorescence properties of curcumin-loaded nanoparticles for cell tracking. Int. J. Nanomedicine 13, 58235836 (2018).

76. Mondal, S. \& Ghosh, S. Role of curcumin on the determination of the critical micellar concentration by absorbance, fluorescence and fluorescence anisotropy techniques. J. Photochem. Photobiol. B Biol. 115, 9-15 (2012).

77. Sahu, A., Kasoju, N., Goswami, P. \& Bora, U. Encapsulation of curcumin in Pluronic block copolymer micelles for drug delivery applications. J. Biomater. Appl. 25, 619-639 (2011). 
78. Sahu, A., Bora, U., Kasoju, N. \& Goswami, P. Synthesis of novel biodegradable and self-assembling methoxy poly (ethylene glycol)-palmitate nanocarrier for curcumin delivery to cancer cells. Acta Biomater. 4, 1752-1761 (2008).

79. Archana, D., Dutta, J. \& Dutta, P. Evaluation of chitosan nano dressing for wound healing: Characterization, in vitro and in vivo studies. Int. J. Biol. Macromol. 57, 193-203 (2013).

80. Mi, F.-L. et al. Fabrication and characterization of a sponge-like asymmetric chitosan membrane as a wound dressing. Biomaterials 22, 165-173 (2001).

81. Winter, G. D. Transcutaneous implants: reactions of the skin-implant interface. J. Biomed. Mater. Res. 8, 99-113 (1974).

82. Brandelero, R. P. H., Yamashita, F. \& Grossmann, M. V. E. The effect of surfactant Tween 80 on the hydrophilicity, water vapor permeation, and the mechanical properties of cassava starch and poly (butylene adipate-co-terephthalate)(PBAT) blend films. Carbohydr. Polym. 82, 1102-1109 (2010).

83. Panatdasirisuk, W., Liao, Z., Vongsetskul, T. \& Yang, S. Separation of oil-in-water emulsions using hydrophilic electrospun membranes with anisotropic pores. Langmuir 33, 5872-5878 (2017).

84. Sundaramurthi, D., Krishnan, U. M. \& Sethuraman, S. Electrospun nanofibers as scaffolds for skin tissue engineering. Polym. Rev. 54, 348-376 (2014).

85. Zorofchian Moghadamtousi, S. et al. A review on antibacterial, antiviral, and antifungal activity of curcumin. Biomed Res. Int. 2014, 1-12 (2014)

86. Teow, S.-Y., Liew, K., Ali, S. A., Khoo, A.S.-B. \& Peh, S.-C. Antibacterial action of curcumin against Staphylococcus aureus: a brief review. J. Trop. Med. 2016, 1-10 (2016)

87. Ngwabebhoh, F. A., Erdagi, S. I. \& Yildiz, U. Pickering emulsions stabilized nanocellulosic-based nanoparticles for coumarin and curcumin nanoencapsulations: in vitro release, anticancer and antimicrobial activities. Carbohydr. Polym. 201, 317-328 (2018).

88. Gopal, J., Muthu, M. \& Chun, S.-C. Water soluble nanocurcumin extracted from turmeric challenging the microflora from human oral cavity. Food Chem. 211, 903-909 (2016).

89. Balouiri, M., Sadiki, M. \& Ibnsouda, S. K. Methods for in vitro evaluating antimicrobial activity: a review. J. Pharm. Anal. 6, 71-79 (2016).

90. Keira, S. M., Ferreira, L. M., Gragnani, A., Duarte, I. D. S. \& Santos, I. A. Experimental model for fibroblast culture. Acta Cir. Bras. $19,11-16(2004)$.

91. Moradi, A., Pramanik, S., Ataollahi, F., Kamarul, T. \& Pingguan-Murphy, B. Archimedes revisited: computer assisted microvolumetric modification of the liquid displacement method for porosity measurement of highly porous light materials. Food Anal. Methods 6, 4396-4401 (2014).

92. Naseri-Nosar, M. et al. Cerium oxide nanoparticle-containing poly ( $\varepsilon$-caprolactone)/gelatin electrospun film as a potential wound dressing material: in vitro and in vivo evaluation. Mater. Sci. Eng. C 81, 366-372 (2017).

93. Kaygusuz, H., Uysal, M., Adımcilar, V. \& Erim, F. B. Natural alginate biopolymer montmorillonite clay composites for vitamin B2 delivery. J. Bioact. Compat. Polym. 30, 48-56 (2015).

94. Nafise, A., Ali, M., Sayyed Abolghasem Sajjadi, T. \& Jebrail, M. Modeling and process optimization of electrospinning of chitosancollagen nanofiber by response surface methodology. Mater. Res. Express 5, 1-23 (2018).

95. Nourmohammadi, E. et al. Evaluation of anticancer effects of cerium oxide nanoparticles on mouse fibrosarcoma cell line. J. Cell. Physiol. 234, 4987-4996 (2018).

96. Aranda, A. et al. Dichloro-dihydro-fluorescein diacetate (DCFH-DA) assay: a quantitative method for oxidative stress assessment of nanoparticle-treated cells. Toxicol. In Vitro 27, 954-963 (2013).

\section{Acknowledgement}

M. Darroudi gratefully acknowledge the financial support of this work, which has been provided by Mashhad University of Medical Sciences (Grant \# 940891).

\section{Author contributions}

Narges Fereydouni (Performer of laboratory tasks and author of the main manuscript text), Aida Gholoobi (Antibacterial test consultant), Jebrail Movaffagh and Arash Goodarzi (Scientific consultant), Nafise Amiri and Alireza Hashemzadeh (Grammatical editing), Susan Darroudi (Data analysis), Majid Darroudi (Corresponding author, designed the study).

\section{Competing interests}

The authors declare no competing interests.

\section{Additional information}

Correspondence and requests for materials should be addressed to N.F. or M.D.

Reprints and permissions information is available at www.nature.com/reprints.

Publisher's note Springer Nature remains neutral with regard to jurisdictional claims in published maps and institutional affiliations.

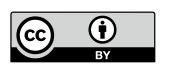

Open Access This article is licensed under a Creative Commons Attribution 4.0 International License, which permits use, sharing, adaptation, distribution and reproduction in any medium or format, as long as you give appropriate credit to the original author(s) and the source, provide a link to the Creative Commons licence, and indicate if changes were made. The images or other third party material in this article are included in the article's Creative Commons licence, unless indicated otherwise in a credit line to the material. If material is not included in the article's Creative Commons licence and your intended use is not permitted by statutory regulation or exceeds the permitted use, you will need to obtain permission directly from the copyright holder. To view a copy of this licence, visit http://creativecommons.org/licenses/by/4.0/.

(C) The Author(s) 2021 